\title{
Non-standard interactions in SMEFT confronted with terrestrial neutrino experiments
}

\author{
Yong Du, ${ }^{a, b}$ Hao-Lin Li, ${ }^{a}$ Jian Tang, ${ }^{c}$ Sampsa Vihonen ${ }^{c}$ and Jiang-Hao $\mathbf{Y u}{ }^{a, d, e, f}$ \\ ${ }^{a}$ CAS Key Laboratory of Theoretical Physics, Institute of Theoretical Physics, \\ Chinese Academy of Sciences, \\ Beijing 100190, P.R. China \\ ${ }^{b}$ Amherst Center for Fundamental Interactions, Physics Department, \\ University of Massachusetts Amherst, \\ Amherst, MA 01003 U.S.A. \\ ${ }^{c}$ School of Physics, Sun Yat-sen University, \\ Guangzhou 510275, China \\ ${ }^{d}$ School of Physical Sciences, University of Chinese Academy of Sciences, \\ Beijing 100049, P.R. China \\ ${ }^{e}$ School of Fundamental Physics and Mathematical Sciences, \\ Hangzhou Institute for Advanced Study, UCAS, \\ Hangzhou 310024, China \\ ${ }^{f}$ International Centre for Theoretical Physics Asia-Pacific, \\ Beijing/Hangzhou, China \\ E-mail: yongdu@umass.edu, lihaolin@itp.ac.cn, \\ tangjian5@mail.sysu.edu.cn, sampsa@mail.sysu.edu.cn, jhyu@itp.ac.cn
}

ABSTRACT: The Standard Model Effective Field Theory (SMEFT) provides a systematic and model-independent framework to study neutrino non-standard interactions (NSIs). We study the constraining power of the on-going neutrino oscillation experiments $\mathrm{T} 2 \mathrm{~K}, \mathrm{NO} \nu \mathrm{A}$, Daya Bay, Double Chooz and RENO in the SMEFT framework. A full consideration of matching is provided between different effective field theories and the renormalization group running at different scales, filling the gap between the low-energy neutrino oscillation experiments and SMEFT at the UV scale. We first illustrate our method with a topdown approach in a simplified scalar leptoquark model, showing more stringent constraints from the neutrino oscillation experiments compared to collider studies. We then provide a bottom-up study on individual dimension-6 SMEFT operators and find NSIs in neutrino experiments already sensitive to new physics at $\sim 20 \mathrm{TeV}$ when the Wilson coefficients are fixed at unity. We also investigate the correlation among multiple operators at the UV scale and find it could change the constraints on SMEFT operators by several orders of 
magnitude compared with when only one operator is considered. Furthermore, we find that accelerator and reactor neutrino experiments are sensitive to different SMEFT operators, which highlights the complementarity of the two experiment types.

Keywords: Effective Field Theories, Neutrino Physics

ArXiv EPRINT: 2011.14292 


\section{Contents}

1 Introduction 1

2 Parameterization of neutrino NSIs 4

3 SMEFT contributions to neutrino NSI parameters 6

4 A brief description of the neutrino oscillation data and the resulting $\begin{array}{ll}\text { constraints to NSI parameters } & 10\end{array}$

$\begin{array}{lll}4.1 & \text { LBL neutrino experiments } \mathrm{T} 2 \mathrm{~K} \text { and } \mathrm{NO} \nu \mathrm{A} & 10\end{array}$

$\begin{array}{lll}4.1 .1 & \text { The T2K facility } & 10\end{array}$

4.1.2 The $\mathrm{NO} \nu \mathrm{A}$ facility 11

4.2 Reactor antineutrino experiments Daya Bay, Double Chooz and RENO 11

4.3 Numerical analysis 13

$\begin{array}{lll}4.4 & \text { Constraining NSI parameters in neutrino experiments } & 15\end{array}$

5 A UV example: the simplified scalar leptoquark model 16

5.1 Model setup and connection to neutrino oscillation experiments 16

$\begin{array}{ll}5.2 \text { Constraints on } M \text { with } \lambda_{L, R}=1 & 18\end{array}$

$\begin{array}{lll}5.3 \text { Constraints on } \lambda_{L, R} \text { with fixed } M & 19\end{array}$

6 Constraints on the dimension-6 SMEFT operators from neutrino oscillation experiments

6.1 Constraints on a single operator 24

$\begin{array}{lll}6.2 & \text { Constraints on multiple operators } & 29\end{array}$

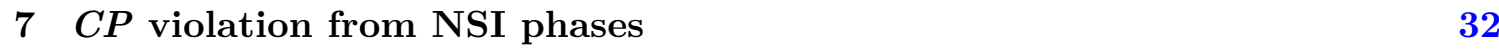

8 Conclusions $\quad 34$

A LEFT for muon decay and the matching between QFT and QM formalisms

\section{Introduction}

The discovery of neutrino oscillations more than two decades ago [1, 2] began an era where the understanding of the neutrino properties has improved at an accelerating pace. Not only have the majority of the neutrino oscillation parameters describing the oscillation of $\nu_{e}$, $\nu_{\mu}$ and $\nu_{\tau}$ been measured to a relatively high precision, but the recent observations from the long-baseline (LBL) accelerator experiments have also hinted that these oscillations may indicate $C P$ violation in the leptonic sector (see ref. [3] for a review). The standard theory of neutrino oscillations has been tested in a variety of neutrino oscillation experiments, which have provided a wealth of data using different neutrino sources and measurement 
techniques. The present generation of on-going neutrino oscillation experiments is already starting to show the level of precision at which probes for non-standard neutrino physics can be started using the novel neutrino oscillation data.

There have been several attempts to look for signals of the physics beyond the Standard Model (BSM) in the neutrino sector [4-12]. While many studies have interpreted the consequences of UV-complete models in the neutrino oscillations through the mixing with the heavy right-handed neutrinos, a great deal of discussion has been placed on the possibility of non-standard interactions (NSIs) influencing neutrino oscillations [3, 13, 14]. The NSI of neutrinos is typically discussed in the context of the ad-hoc parameterization of effective couplings $\epsilon^{s}, \epsilon^{d}[15-18]$ and $\epsilon^{m}$ [19], which describe the non-standard effects in the source, detection and propagation, respectively. The majority of the literature focused on finding the constraints on each parameter in neutrino oscillation experiments, but new physics has so far been mainly treated as uncorrelated energy-independent parameters. The efforts to connect the knowledge into a full UV-complete model have altogether been fairly limited. There is now a growing demand for a systematic treatment that connects the low-energy BSM phenomenology of the neutrino NSI to the high-scale regime.

The Standard Model Effective Fields Theory (SMEFT) provides a systematic and model-independent way to parameterize the effects of some heavy degrees of freedom in the UV theory into Wilson coefficients of a set of higher dimensional operators. These operators are constructed by the SM fields and respect the SM gauge symmetries. Therefore, the SMEFT is very robust to study the effects of some UV physics on neutrino NSI parameters. A schematic workflow to find relations between the parameters in the UV model and the neutrino NSI parameters is shown in figure 1. Generally, in the top-down point of view, the Wilson coefficients of the SMEFT operators can be derived as a function of parameters in the UV theory by integrating out the heavy fields at the UV scale $\Lambda$. This procedure of deriving the relations between the Wilson coefficients and the couplings in the UV model is called matching, and is indicated by the first row of figure 1. Note that the Wilson coefficients after this matching are defined at the UV scale $\Lambda$, while current experiments are performed at a much lower scale. For example, observables from collider experiments are usually defined at the weak scale $m_{W}$, thus the renormalization group equation (RGE) running of these Wilson coefficients needs to be considered to correctly find contributions to these observables from the UV theory. This step is represented by the arrow connecting the purple and the green in figure 1.

On the other hand, neutrino oscillation experiments are performed at an even lower scale, and theoretically, the low energy effective field theory (LEFT) around $2 \mathrm{GeV}$ stands as an ideal framework for the study. Obviously, there exists an energy gap between the SMEFT after the aforementioned RGE running and this LEFT. Therefore, extra matching and running need to be appropriately done before making a correct and meaningful prediction for the neutrino NSI parameters from the UV theory. These extra steps are indicated by the last two rows of figure 1, where one firstly translates the SMEFT at the weak scale into the LEFT through matching as shown by the arrow connecting the two greens. This second matching is achieved by breaking the electroweak symmetry and integrating out the top quark, the Higgs boson and the massive gauge bosons. Note that 


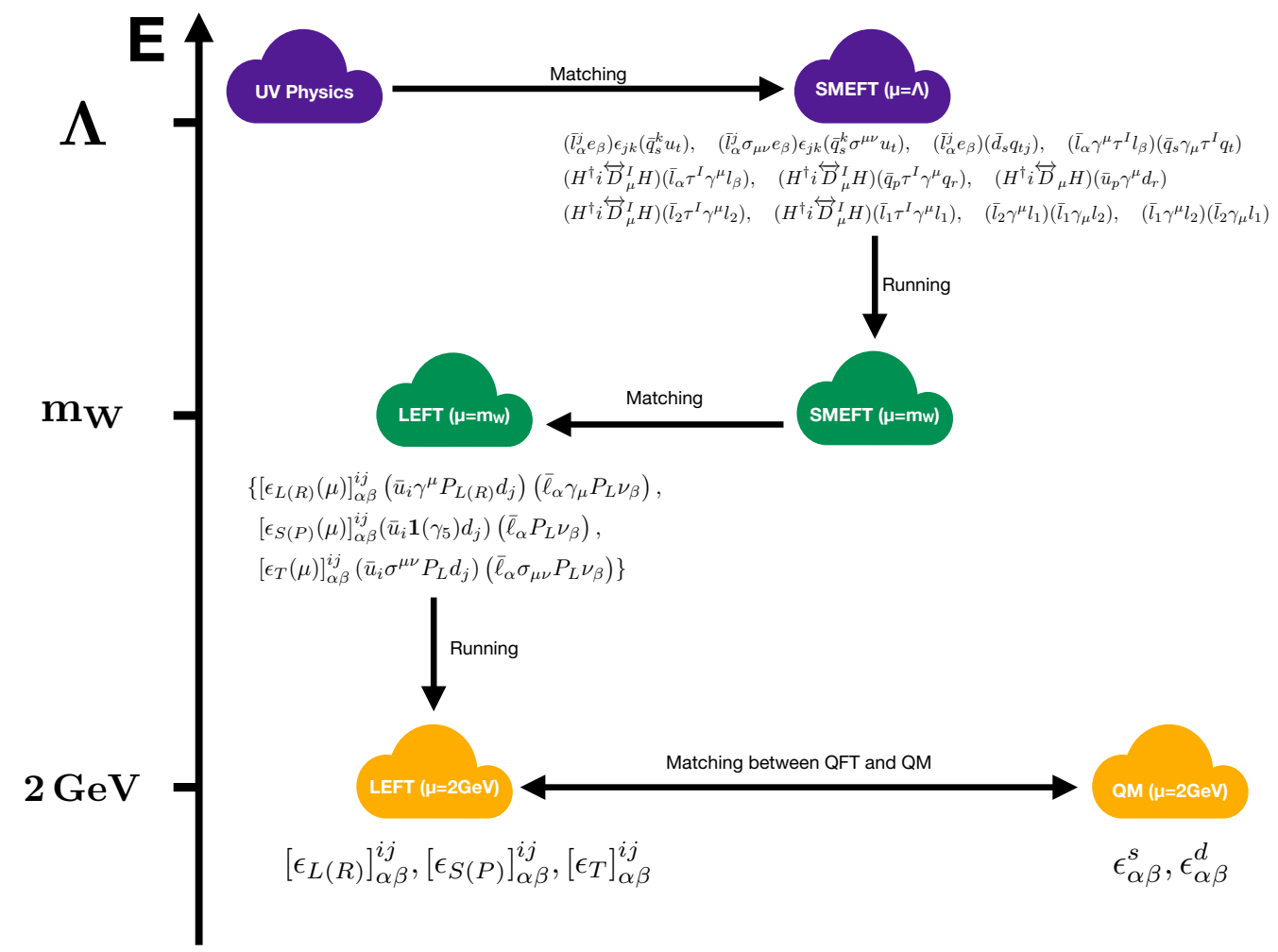

Figure 1. Schematic description of relating the UV parameters to the neutrino NSI parameters. The vertical axis defines the scale where corresponding EFTs are present. The enumerated operators and/or Wilson coefficients under a certain EFT name at some energy scale $\mu$ are those relevant for our study in this work. See the main text for the details.

all the Wilson coefficients in the LEFT after this step are defined at the weak scale $m_{W}$. Then secondly, one applies the RGE and runs these Wilson coefficients down to the $2 \mathrm{GeV}$ scale for neutrino oscillation experiments. This is indicated by the arrow connecting the green and the yellow in figure 1. Now the conventional neutrino NSI parameters in the quantum field theory (QFT) description are encoded in the Wilson coefficients $\epsilon_{L, R, S, P, T}$ of the several charged current operators in LEFT as shown in figure 1 and eq. (2.3) at the $2 \mathrm{GeV}$ scale. From there on, we leave out the scale parameter $\mu$ and unless otherwise specified, $\epsilon_{L, R, S, P, T}$ shall all be understood as parameters defined at the $2 \mathrm{GeV}$ scale. Finally, these Wilson coefficients can be matched to the NSI parameters $\epsilon^{s, d}$ in the quantum mechanic (QM) formalism. A faithful matching of these two types of neutrino NSI parameters only exist when the event rates calculated in the QFT formalism are expanded to linear order in the NSI parameters. This point was recently presented in ref. [20] and is represented by the arrow connecting the two yellows in our schematic plot figure 1 .

In the present work, we make an effort to systematically study the connection between terrestrial neutrino oscillation experiments and the high-energy phenomenology. We study the physical potential of the neutrino oscillation experiments by reflecting on the recent findings of the LBL accelerator experiments Tokai-to-Kamioka (T2K) $[21,22]$ and NuMI 
Off-axis neutrino Appearance $(\mathrm{NO} \nu \mathrm{A})[23,24]$ and the reactor experiments Daya Bay [2527], Double Chooz [28, 29] and Reactor Experiment for Neutrino Oscillation (RENO) [30, 31]. Practically, we use the Wilson package [32] to take care of the running from the scale $\Lambda$ down to $2 \mathrm{GeV}$, as well as the second matching between SMEFT and the LEFT at $\mu=m_{W}$ shown in figure 1. ${ }^{1}$ The first matching is model dependent and is thus usually worked out manually model by model. We will discuss more about it in section 2 and provide an example in section 5 for illustration. The last matching is universal and has been studied in ref. [20] recently. The NSI parameters acquired this way serve as our input to the General Long-Baseline Experiment Simulator (GLoBES) [35, 36] through its new physics extension [37].

This article is decomposed into the following sections: in section 2 we review the standard parameterization of the NSIs in neutrino oscillations and summarize the matching between the QFT and the QM formalisms relevant for our study. Section 3 focuses on the connection between the SMEFT and the neutrino NSI parameters. We describe the neutrino oscillation data and the related analysis methods used in this work in section 4 . Then we present a UV example, i.e., the simplified leptoquark model in section 5 to illustrate our approach following the workflow of figure 1 . We finally present a systematical analysis on the dominant dimension-6 SMEFT operators by providing constraints on the UV scale $\Lambda$ and the Wilson coefficients from neutrino experiments in section 6. The implications on the $C P$ violation in the leptonic sector are briefly discussed in section 7 . The results are then summarized in section 8.

\section{Parameterization of neutrino NSIs}

Since neutrino oscillations are low-energy experiments, the Quantum Mechanical (QM) formalism is often used to compute the oscillation probability $P_{\alpha \beta}=\left|\left\langle\nu_{\alpha}^{d}\left|e^{-i H L}\right| \nu_{\beta}^{s}\right\rangle\right|^{2}$, which describes the probability of a source neutrino of flavor $\beta$ being detected as a neutrino of flavor $\alpha$. The meaning of "flavor" here is modified according to the inclusion of the non-standard interactions and is different from the original definition of the flavor that is associated with the lepton doublets diagonalizing the charged lepton mass matrix. The relation between these two flavor eigenstates are related by the QM production and detection NSI parameters $\epsilon^{s}$ and $\epsilon^{d}[15-18]$ :

$$
\left|\nu_{\alpha}^{s}\right\rangle=\frac{\left(1+\epsilon^{s}\right)_{\alpha \gamma}}{N_{\alpha}^{s}}\left|\nu_{\gamma}\right\rangle, \quad\left\langle\nu_{\beta}^{d}\right|=\left\langle\nu_{\gamma}\right| \frac{\left(1+\epsilon^{d}\right)_{\gamma \beta}}{N_{\beta}^{d}},
$$

where $N_{\alpha}^{s}=\sqrt{\left[\left(1+\epsilon^{s}\right)\left(1+\epsilon^{s \dagger}\right)\right]_{\alpha \alpha}}$ and $N_{\beta}^{d}=\sqrt{\left[\left(1+\epsilon^{d \dagger}\right)\left(1+\epsilon^{d}\right)\right]_{\beta \beta}}$. The repeated indices $\alpha$ and $\beta$ on the right hand side of equations refer to the diagonal elements, and are not to be confused with indices contraction. The information about matter effects affecting the propagation of the neutrino oscillation in media is encoded in the Hamiltonian $H$ :

$$
H=\frac{1}{2 E_{\nu}}\left[U\left(\begin{array}{ccc}
0 & 0 & 0 \\
0 & \Delta m_{21}^{2} & 0 \\
0 & 0 & \Delta m_{31}^{2}
\end{array}\right) U^{\dagger}+A\left(\begin{array}{ccc}
1+\varepsilon_{e e}^{m} & \epsilon_{e \mu}^{m} & \epsilon_{e \tau}^{m} \\
\epsilon_{e \mu}^{m *} & \epsilon_{\mu \mu}^{m} & \epsilon_{\mu \tau}^{m} \\
\epsilon_{e \tau}^{m *} & \epsilon_{\mu \tau}^{m *} & \epsilon_{\tau \tau}^{m}
\end{array}\right)\right],
$$

\footnotetext{
${ }^{1}$ For the matching and running between SMEFT and LEFT for charge-current interactions, see refs. [33, 34].
} 


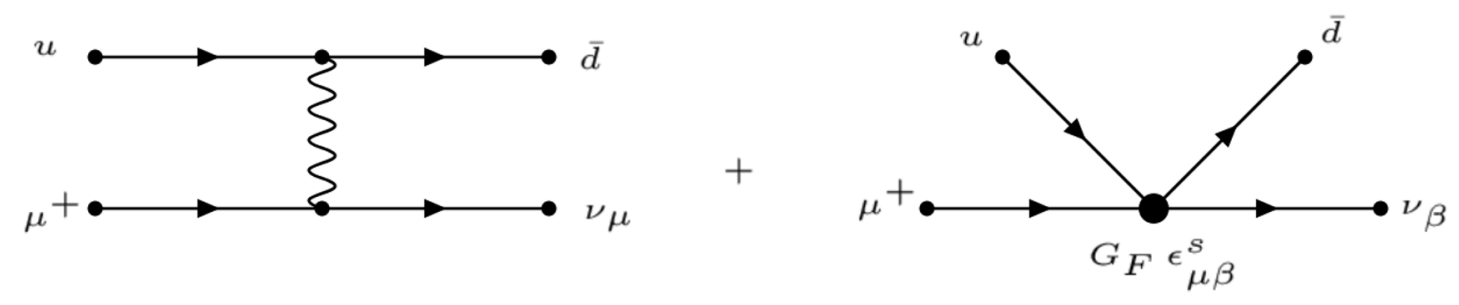

Figure 2. An illustration of the non-standard interactions in the neutrino source. A positively charged pion decays incoherently into a positive muon and a muon neutrino or a different flavor neutrino.

where $U$ is the Pontecorvo-Maki-Nakagawa-Sakata matrix [38-42] and $A=\sqrt{2} G_{F} N_{e}$, with $G_{F}$ denoting the Fermi constant and $N_{e}$ the electron number density in the medium, defines the standard matter potential [19, 43]. The matrix elements $\epsilon_{\alpha \beta}^{m}(\alpha, \beta=e, \mu, \tau)$ are the matter NSI parameters. A schematic illustration of the source NSI is presented in figure 2, where the pion decay process is influenced by non-standard neutrino interactions.

The NSI parameters defined in the QM formalism have been widely used in the study of neutrino oscillations, and their concepts are rather phenomenological. However, to study the effects originating from some UV physics, it is better to parameterize the NSI parameters in the QFT framework, i.e., in terms of the Wilson coefficients in the Lagrangian of the LEFT since these Wilson coefficients are directly related to the parameters in the UV theory as depicted in figure 1. On the other hand, it was recently pointed out in ref. [20] that matching the differential rate $R_{\alpha \beta}$, defined as the number of oscillation events observed per second per neutrino energy [20, 44], in both formalisms to obtain the connection between the NSI parameters and the Wilson coefficients in LEFT is highly non-trivial. Furthermore, this matching procedure is not guaranteed to give a meaningful match of the two sets of parameters unless one works in the linear approximation in terms of $\epsilon^{s, d}$. In what follows, in light of the smallness of the neutrino NSI parameters, we will adopt the matching procedure described in ref. [20] to the linear order and study effects from these charge-current NSI parameters. ${ }^{2}$

To start, we write down the most general charged-current (CC) neutrino NSIs in LEFT as $[20]:^{3}$

$$
\begin{aligned}
\mathcal{L}_{\mathrm{CC}} \supset & -\frac{2 V_{u d}}{v^{2}}\left\{\left[\mathbf{1}+\epsilon_{L}\right]_{\alpha \beta}^{i j}\left(\bar{u}_{i} \gamma^{\mu} P_{L} d_{j}\right)\left(\bar{\ell}_{\alpha} \gamma_{\mu} P_{L} \nu_{\beta}\right)+\left[\epsilon_{R}\right]_{\alpha \beta}^{i j}\left(\bar{u}_{i} \gamma^{\mu} P_{R} d_{j}\right)\left(\bar{\ell}_{\alpha} \gamma_{\mu} P_{L} \nu_{\beta}\right)\right. \\
& +\frac{1}{2}\left[\epsilon_{S}\right]_{\alpha \beta}^{i j}\left(\bar{u}_{i} d_{j}\right)\left(\bar{\ell}_{\alpha} P_{L} \nu_{\beta}\right)-\frac{1}{2}\left[\epsilon_{P}\right]_{\alpha \beta}^{i j}\left(\bar{u}_{i} \gamma_{5} d_{j}\right)\left(\bar{\ell}_{\alpha} P_{L} \nu_{\beta}\right) \\
& \left.+\frac{1}{4}\left[\epsilon_{T}\right]_{\alpha \beta}^{i j}\left(\bar{u}_{i} \sigma^{\mu \nu} P_{L} d_{j}\right)\left(\bar{\ell}_{\alpha} \sigma_{\mu \nu} P_{L} \nu_{\beta}\right)+\text { h.c. }\right\}
\end{aligned}
$$

\footnotetext{
${ }^{2}$ For the neutral-current neutrino NSIs, see, for example, ref. [14] for a recent review and refs. [45-51] for their recent studies. In this work, however, we will only focus on the charged-current NSI parameters.

${ }^{3}$ For this CC NSIs, we adopt the convention used in ref. [20], where the (V+A), scalar, pseudoscalar and tensor-type interactions are also introduced. In the case of general neutrino interactions, we refer the reader to ref. [52].
} 
where from now on we use the Roman letters $i, j=\{1,2,3\}$ to represent the flavor of quarks, and the Greek letters $\alpha, \beta=\{e, \mu, \tau\}$ to that of charged leptons and neutrinos, $P=P_{L, R}$ are the chiral projection operators for the left- and the right-handed state of Dirac spinors. Since the energy scale of neutrino oscillation experiments are oftentimes very small, the first generation of quarks is to be understood when the $i, j$ indices are omitted for the NSI parameters $\epsilon_{L, R, S, P, T}$.

Starting from eq. (2.3) and following the matching procedure of ref. [20], we reproduce the following matching formulas originally derived in ref. [20] to the linear order of $\epsilon_{\alpha \beta}^{s, d}$, $:^{4}$

$$
\begin{aligned}
\epsilon_{e \beta}^{s} & =\left[\epsilon_{L}-\epsilon_{R}-\frac{g_{T}}{g_{A}} \frac{m_{e}}{f_{T}\left(E_{\nu}\right)} \epsilon_{T}\right]_{e \beta}^{*}, \quad(\beta \text { decay) } \\
\epsilon_{\beta e}^{d} & =\left[\epsilon_{L}+\frac{1-3 g_{A}^{2}}{1+3 g_{A}^{2}} \epsilon_{R}-\frac{m_{e}}{E_{\nu}-\Delta}\left(\frac{g_{S}}{1+3 g_{A}^{2}} \epsilon_{S}-\frac{3 g_{A} g_{T}}{1+3 g_{A}^{2}} \epsilon_{T}\right)\right]_{e \beta}, \text { (inverse } \beta \text { decay) } \\
\epsilon_{\mu \beta}^{s} & =\left[\epsilon_{L}-\epsilon_{R}-\frac{m_{\pi}^{2}}{m_{\mu}\left(m_{u}+m_{d}\right)} \epsilon_{P}\right]_{\mu \beta}^{*}, \quad \text { (pion decay) }
\end{aligned}
$$

where $g_{S}=1.022(10), g_{A}=1.251(33)$ and $g_{T}=0.987(55)$ are the scalar, axial-vector and tensor charges of the nucleon [53-56], $\Delta \equiv m_{n}-m_{p} \simeq 1.3 \mathrm{MeV}, E_{\nu}$ is the neutrino energy, and $f_{T}\left(E_{\nu}\right)$ is the nucleon form factor resulting from the tensor-type NSI in eq. (2.3), for which we use the same parameterization as that in ref. [57]. Note that for the inverse $\beta$ decay, the formula above only includes the Gamow-Teller type interactions since reactor beta transitions like Daya Bay, Double Chooz and RENO discussed above, are mostly of this type [58]. On the other hand, one may also expect contributions to $\epsilon_{e \beta}^{s}$ from muon decay, however, as we will discuss in the next section, NSI effects from muon decay will be absorbed into the definition of the Fermi constant in our setup.

Before discussing the effects from the CC NSIs, we would like to emphasize that whenever matching is involved, one shall note that all the parameters in eq. (2.3) are to be understood as parameters defined at the $2 \mathrm{GeV}$ scale. Therefore, when one studies the effects of some new physics that lives at a much higher scale, the running effects, as illustrated in figure 1 , shall be included as they could contribute significantly to these parameters due to the large QCD coupling. A complete study of the running effects is provided in ref. [59]. In our numerical analysis, we count on the Wilson package [32] to take care of these effects.

\section{SMEFT contributions to neutrino NSI parameters}

Since no new particles have been observed at the LHC since 2012 after the discovery of the Higgs, SMEFT has become a popular toolset to systematically study potential deviations from SM predictions due to new physics residing in an energy scale $\Lambda$ much higher than the weak scale. Hence, it is thus natural to study the effects on neutrino NSIs from this new physics in the framework of SMEFT. To be specific, we shall derive constraints on the SMEFT Wilson coefficients from neutrino oscillation experiments following the workflow in figure 1 and using eqs. (2.4)-(2.6) for the matching that essentially relates the UV

\footnotetext{
${ }^{4}$ Here, we use the same notation for the nucleon tensor form factor $g_{T}$ as in ref. [20] in eqs. (2.4)-(2.5).
} 
parameters to $\epsilon^{s, d}$. Similar work focusing on the neutral-current source NSI can be found in ref. [60], though no source and detection NSI were taken into account. Ref. [61] studied charge-current NSIs, but they only considered the lepton flavor conserving part of the NSI parameters in eq. (2.3). In this section, we will describe the matching framework we use to derive our results. Our study follows the results in ref. [62] and is implemented in the Python package Wilson.

In general, the SMEFT can be organized by the dimension of the operators:

$$
\mathcal{L}_{\mathrm{SMEFT}}=\sum_{D} \sum_{i_{D}} \frac{c_{i_{D}}}{\Lambda^{D-4}} \mathcal{O}_{i}^{D},
$$

where $D$ denotes the dimensions of the operators starting from $5, i_{D}$ labels the effective operators for a given dimension $D, c_{i_{D}}$ is the dimensionless Wilson coefficient, $\Lambda$ represents the characteristic UV scale. In this work, we will focus on the dimension-6 operators only and assume that neutrino masses are generated by the dimension-5 Weinberg operator [63].

Starting from a certain UV theory around scale $\Lambda$, its contributions to SMEFT operators can be readily obtained by integrating out the heavy fields with the covariant derivative expansion (CDE) techniques [64-66]. Alternatively, one can calculate the amplitudes in both the UV theory and the SMEFT, and then obtain their relations through amplitudes matching. See, for example, ref. [67] for a detailed discussion. This is a paradigm of the top-down EFT approach in studying the phenomenology of new physics, depicted by the first row of figure 1. We will show a concrete example of this approach to study constraints from neutrino oscillations on a simplified scalar leptoquark model in section 5. On the other hand, in the bottom-up EFT approach, one can view the SMEFT at an arbitrary new physics scale as the starting point and treat all the Wilson coefficients as independent parameters. This approach is more general while the correlation among the Wilson coefficients is lost. In section 6 , through a careful study on the correlation of dimension- 6 SMEFT operators for neutrino oscillation experiments, we will demonstrate this correlation is in general important.

In both the EFT approaches described above, the Wilson coefficients of the SMEFT operators are all defined at the new physics scale $\Lambda$, while a natural matching between SMEFT and LEFT is around $m_{W}$, where one integrates out $t$ quark, the Higgs boson, and the $W, Z$ bosons in the SM as implied by the second row of figure 1 . Therefore, before the matching procedure is done, one needs to run the SMEFT Wilson coefficients from the scale $\Lambda$ down to $m_{W}$, i.e., from the first to the second row of figure 1 . This running of the SMEFT Wilson coefficients were thoroughly studied in refs. [68-70]. Due to the running effects, neutral current interactions in the UV model can finally generate charged current NSIs. Taking the minimal $Z^{\prime}$ model as an example, we assume that the SM gauge group is extended by a $\mathrm{U}(1)_{z}$ associated with the new gauge boson $Z^{\prime}$ and a complex scalar $\phi$ which is responsible for the spontaneous breaking of the $\mathrm{U}(1)_{z}$ giving mass to $Z^{\prime}$. With no new fermion fields introduced, the gauge anomaly cancellation restricts the $\mathrm{U}(1)_{z}$ charges of the SM fields to be proportional to their $\mathrm{U}(1)_{Y}$ hypercharges or trivially to equal to zero. In this case we find that a tree level matching generates flavor conserving neutral 
current $C_{e е \mu \mu}^{l l}$, which generates $C_{e \mu \mu e}^{l l}$ through the RGE running and further induces the $\epsilon_{L}$ via the calibration of $G_{F}$ as we will discuss below.

The matching between SMEFT and the LEFT is studied in detail in ref. [62]. At tree level, we classify the SMEFT operators that contribute to the neutrino NSI operators in the LEFT after matching into two types as listed below, where the type-A ones generates the operators in the LEFT directly via expansions of the left-handed lepton and the quark doublets, and the type- $\mathrm{B}$ ones contribute by modifying the interactions between the gauge bosons and fermions after setting the Higgs field to its vacuum expectation value $v_{T}$ and integrating out the $W$ bosons and setting.

\begin{tabular}{|c|cc|}
\hline Type-A & $\mathcal{O}_{\text {lequ }}^{(1)}=\left(\bar{l}_{\alpha}^{j} e_{\beta}\right) \epsilon_{j k}\left(\bar{q}_{s}^{k} u_{t}\right)$ & $\mathcal{O}_{l e q u}^{(3)}=\left(\bar{l}_{\alpha}^{j} \sigma_{\mu \nu} e_{\beta}\right) \epsilon_{j k}\left(\bar{q}_{s}^{k} \sigma^{\mu \nu} u_{t}\right)$ \\
& $\mathcal{O}_{l e d q}=\left(\bar{l}_{\alpha}^{j} e_{\beta}\right)\left(\bar{d}_{s} q_{t j}\right)$ & $\mathcal{O}_{l q}^{(3)}=\left(\bar{l}_{\alpha} \gamma^{\mu} \tau^{I} l_{\beta}\right)\left(\bar{q}_{s} \gamma_{\mu} \tau^{I} q_{t}\right)$ \\
\hline \multirow{4}{*}{ Type-B } & $\mathcal{O}_{H l}^{(3)}=\left(H^{\dagger} i \overleftrightarrow{D}_{\mu}^{I} H\right)\left(\bar{l}_{\alpha} \tau^{I} \gamma^{\mu} l_{\beta}\right)$ & $\mathcal{O}_{H q}^{(3)}=\left(H^{\dagger} i \overleftrightarrow{D}_{\mu}^{I} H\right)\left(\bar{q}_{p} \tau^{I} \gamma^{\mu} q_{r}\right)$ \\
& $\mathcal{O}_{H u d}=\left(H^{\dagger} i \overleftrightarrow{D}_{\mu} H\right)\left(\bar{u}_{p} \gamma^{\mu} d_{r}\right) \quad \mathcal{O}_{2112}^{l l}=\left(\bar{l}_{2} \gamma^{\mu} l_{1}\right)\left(\bar{l}_{1} \gamma_{\mu} l_{2}\right)$ \\
$\mathcal{O}_{1221}^{l l}=\left(\bar{l}_{1} \gamma^{\mu} l_{2}\right)\left(\bar{l}_{2} \gamma_{\mu} l_{1}\right)$ & $\mathcal{O}_{H l}^{(3)}=\left(H^{\dagger} i \overleftrightarrow{D}_{\mu}^{I} H\right)\left(\bar{l}_{2} \tau^{I} \gamma^{\mu} l_{2}\right)$ \\
& $\mathcal{O}_{H l}^{(3)}=\left(H^{\dagger} i \overleftrightarrow{D}_{\mu}^{I} H\right)\left(\bar{l}_{1} \tau^{I} \gamma^{\mu} l_{1}\right)$ \\
11
\end{tabular}

One example of the type-A operators is:

$$
\mathcal{O}_{l e q u}^{(1)}=\bar{l}_{\alpha}^{j} e_{\beta} \epsilon_{j k} \bar{q}_{s}^{k} u_{t}
$$

where $l_{\alpha}=\left(\nu_{L, \alpha}, \ell_{L, \alpha}\right)^{T}$ and $q_{s}=\left(u_{L, s}, d_{L, s}\right)^{T}$ are the $\mathrm{SU}(2)_{L}$ lepton and quark doublets. Expanding the conjugate of this operator, one can find that it contains the following $\mathrm{CC}$ neutrino NSI relevant operators:

$$
\bar{l}_{\alpha}^{j} e_{\beta} \epsilon_{j k} \bar{q}_{s}^{k} u_{t} \supset\left(\bar{u}_{t} P_{L} d_{s}\right)\left(\bar{\ell}_{\alpha} P_{L} \nu_{\beta}\right)=\frac{1}{2}\left(\bar{u}_{t} d_{s}\right)\left(\bar{\ell}_{\alpha} P_{L} \nu_{\beta}\right)-\frac{1}{2}\left(\bar{u}_{t} \gamma^{5} d_{s}\right)\left(\bar{\ell}_{\alpha} P_{L} \nu_{\beta}\right)
$$

Hence, if the corresponding dimensionless Wilson coefficients of the operators in eq. (3.2)

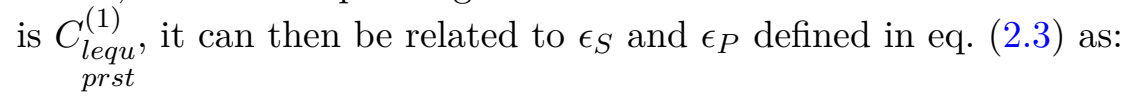

$$
\left(\epsilon_{S}\right)_{\alpha \beta}^{t s}=\left(\epsilon_{P}\right)_{\alpha \beta}^{t s}=(\underset{\alpha \beta s t}{(1)})^{*}
$$

For the Type-B operators, one can parameterize their effects in the following Lagrangian [62]:

$$
\mathcal{L}_{\text {gauge }}=-\frac{\bar{g}_{2}}{\sqrt{2}}\left\{\mathcal{W}_{\mu}^{+} j_{\mathcal{W}}^{\mu}+\text { h.c. }\right\}-\bar{g}_{Z} Z_{\mu} j_{Z}^{\mu},
$$

where once again we only focus on the $\mathrm{CC} j_{W}^{\mu}$ :

$$
j_{\mathcal{W}}^{\mu}=\left[W_{l}\right]_{\alpha \beta} \bar{\nu}_{L \alpha} \gamma^{\mu} e_{L \beta}+\left[W_{q}\right]_{p r} \bar{u}_{L p} \gamma^{\mu} d_{L r}+\left[W_{R}\right]_{p r} \bar{u}_{R p} \gamma^{\mu} d_{R r}
$$


with $W_{l, q, R}$ parameterizing the modification of the $W$ boson coupling to the corresponding fermion currents

$$
\left[W_{l}\right]_{\alpha \beta}=\left[\delta_{\alpha \beta}+v_{T}^{2} C_{\alpha \beta}^{(3)}\right], \quad\left[W_{q}\right]_{p r}=\left[\begin{array}{r}
\delta_{p r}+v_{T}^{2} C_{H_{p r}}^{(3)}
\end{array}\right], \quad\left[W_{R}\right]_{p r}=\left[\frac{1}{2} v_{T}^{2} C_{p r} \underset{p r}{ }\right]
$$

The $W$ boson mass is $M_{\mathcal{W}}=\bar{g}_{2}^{2} v_{T}^{2} / 4$, with $\bar{g}_{2}$ the modified gauge coupling of the $\mathrm{SU}(2)$ group when normalization of the gauge fields due to the presence of $\mathcal{O}_{H W}=$ $|H|^{2} W_{\mu \nu}^{I} W^{I \mu \nu}$ [62]. However, as we shall see immediately, $\mathcal{O}_{H W}$ is not relevant for our tree-level matching. After integrating out the $W$ boson at tree level, the effective Lagrangian can be written as:

$$
\mathcal{L}_{\mathrm{C}}=-\frac{\bar{g}_{2}^{2}}{2 M_{\mathcal{W}}} j_{\mathcal{W}}^{\mu} j_{\mathcal{W} \mu}=-\frac{2}{v_{T}^{2}} j_{\mathcal{W}}^{\mu} j_{\mathcal{W} \mu}
$$

note that $\bar{g}_{2}$ cancels in the numerator and the denominator. Upon expanding $j_{\mathcal{W}}^{\mu}$, the above equation will then generate all the terms in eq. (2.3). However, one needs to pay particular attention to the fact that $v_{T}$ above is different from $v$ in eq. (2.3). The latter is defined through the Fermi constant as $G_{F}=1 /\left(\sqrt{2} v^{2}\right)$, and the relation between $v_{T}$ and $G_{F}$, the Fermi constant $G_{\mu}$ obtained from muon decay in our setup, is as follows:

$$
\frac{4 G_{F}}{\sqrt{2}}=\frac{4 G_{\mu}}{\sqrt{2}}=\frac{2}{v_{T}^{2}}-C_{\mu e e \mu}^{l l}-C_{e \mu \mu e}^{l l}+\underset{\mu \mu}{C_{H l}^{(3)}}+C_{e e}^{(3)}
$$

The term on the right hand side is essentially the Wilson coefficients of the LEFT operators $\left(\bar{\nu}_{L \mu} \gamma^{\mu} \nu_{L e}\right)\left(\bar{e}_{L} \gamma_{\mu} \mu_{L}\right)$ after the tree level matching. In principle, there could be a non-vanishing Wilson coefficients of $\left(\bar{\nu}_{L \mu} \gamma^{\mu} \nu_{L e}\right)\left(\bar{e}_{R} \gamma_{\mu} \mu_{R}\right)$ from the new physics effects contributing to the muon decay process, while its contribution is higher order or suppressed by the $m_{e} / m_{\mu}$ in the interference terms due to the opposite chirality of the electron in the two operators [62]. Since there are two neutrinos involved in muon decay, the matching between the QFT and the QM formalisms are less trivial compared with pion decay, beta and inverse beta decay discussed in section 2. On the other hand, for different setups with respect to $G_{F}$ as discussed above, it would become necessary to have the matching formulae for muon decay. For this reason, we present these matching formulae in appendix A. Our results agree with those in ref. [20] for both neutrinos in the final states after Taylor expansion around small electron mass $m_{e}$. A comparison between our results and those in ref. [20] is also discussed in appendix A.

The last subtlety of this framework is related to the definition of quark flavors in operators. At tree level, the mass matrices $\left[M_{\psi}\right]_{r s}$ with SMEFT dimension-6 operators are:

$$
\left[M_{\psi}\right]_{r s}=\frac{v_{T}}{\sqrt{2}}\left(\left[Y_{\psi}\right]_{r s}-\frac{1}{2} v^{2} C_{\psi H}^{*}\right), \quad \psi=u, d .
$$

Depending on whether the up-type quark or the down-type quark mass matrix is diagonal, there are two types of bases at the definition scale, i.e., the "Warsaw up" and the "Warsaw down" bases:

$$
\begin{array}{rll}
\text { Warsaw up : } & M_{d} \rightarrow \operatorname{diag}\left(m_{d}, m_{s}, m_{b}\right) V^{\dagger}, & M_{u} \rightarrow \operatorname{diag}\left(m_{u}, m_{c}, m_{t}\right), \\
\text { Warsaw down : } & M_{d} \rightarrow \operatorname{diag}\left(m_{d}, m_{s}, m_{b}\right), & M_{u} \rightarrow V^{\dagger} \operatorname{diag}\left(m_{u}, m_{c}, m_{t}\right) .
\end{array}
$$


For the lepton sector, in either the "Warsaw up" or the "Warsaw down" basis, we always choose the bases of the lepton doublets and the right-handed charged lepton fields such that the mass matrix of the charged leptons is diagonal. We would like to emphasize here that both bases are specifically defined at a fixed scale such that when running effects are included, off-diagonal elements in the mass matrices are likely to reappear at a different scale. Therefore, whenever a running is performed, one needs to rotate the basis of fermion fields to change the operators back into the "Warsaw up" or the "Warsaw down" basis.

\section{A brief description of the neutrino oscillation data and the resulting constraints to NSI parameters}

The focus of our work is in the present generation of neutrino oscillation experiments and to investigate the effects of dimension-6 SMEFT operators on neutrino oscillation. To this end, we simulate the neutrino oscillation corresponding to the recently collected data in the LBL experiments $\mathrm{T} 2 \mathrm{~K}$ and $\mathrm{NO} \nu \mathrm{A}$ and the reactor experiments Daya Bay, Double Chooz and RENO. We consider the data sets published in the Neutrino 2020 conference [71] and reproduce them with GLoBES. In this section, we briefly describe the nature of the simulated data and numerical methods used in this work.

\subsection{LBL neutrino experiments $\mathrm{T} 2 \mathrm{~K}$ and $\mathrm{NO} \nu \mathrm{A}$}

The LBL neutrino experiments selected for this work are T2K [21] and NO $\nu \mathrm{A}[24]$ experiments. T2K and $\mathrm{NO} \nu \mathrm{A}$ are on-going LBL experiments where intensive beams of muon neutrinos and antineutrinos are created through pion decay. Muon neutrinos and antineutrinos produced via this method make the majority of the beam composition, about $96 \%-98 \%$, while the rest of the beam consists of the intrinsic beam background of electron neutrinos and antineutrinos.

\subsubsection{The T2K facility}

The T2K beam facility is located at J-PARC in Japan, which produces neutrinos and antineutrinos with a proton accelerator. The proton beam generates neutrinos and antineutrinos at $0.77 \mathrm{~kW}$ power output. The beam was originally scheduled to run 2 years in neutrino mode and 6 years in antineutrino mode. The produced muon neutrinos and antineutrinos are sent to traverse $295 \mathrm{~km}$ underground, upon which they undergo oscillations to other flavor states, mainly electron neutrinos and antineutrinos. The T2K collaboration recently reported oscillation data corresponding to $3.13 \times 10^{21}$ protons-on-target (POT) [21, 72] The data consists of the combined run of $1.49 \times 10^{21}$ POT in neutrino beam and $1.64 \times 10^{21}$ POT in antineutrino beam modes in T2K beam between 2009 and 2018.

The far detector of T2K is Super-Kamiokande, which is a large ultra-pure water-based neutrino detector where neutrinos and antineutrinos sent from J-PARC are observed via the creation of Cherenkov rings. The neutrinos undergo charged-current interactions in the detector creating charged leptons of the same flavor. It is these charged particles that lead to the observation of the Cherenkov rings. Super-Kamiokande has 22.5 kton fiducial mass and it stands $2.5^{\circ}$ off the beam axis. In this position, the neutrino and 
antineutrino beams from J-PARC peak at about $600 \mathrm{MeV}$, and the dominant interaction type is therefore the charged-current quasi-elastic (CCQE) interaction with small chance for resonant charged-current pion production $(\mathrm{CC} 1 \pi)$. The neutrino and antineutrino beams are also studied at the ND280 near detector complex, which includes a Water Cherenkov part of $1,529 \mathrm{~kg}$ fiducial mass at $280 \mathrm{~m}$ distance and $2.5^{\circ}$ from the source. The near detector data corresponds to $5.8 \times 10^{20} \mathrm{POT}$ and $3.9 \times 10^{20} \mathrm{POT}$ in neutrino and antineutrino beam modes, respectively

The oscillation data from $\mathrm{T} 2 \mathrm{~K}$ is divided into five distinct samples based on their event topology. The appearance data in T2K probes $\nu_{\mu} \rightarrow \nu_{\mu}$ and $\bar{\nu}_{\mu} \rightarrow \bar{\nu}_{\mu}$ oscillations, whereas the disappearance data consists of events arising from $\nu_{\mu} \rightarrow \nu_{e}$ and $\bar{\nu}_{\mu} \rightarrow \bar{\nu}_{e}$ oscillations undergoing CCQE interaction. The appearance data also has a third sample, where the $\nu_{e}$ are observed together with a positively charged pion in the final state $\left(\nu_{e} \mathrm{CC} 1 \pi^{+}\right)$. The $\nu_{\mu}$ and $\bar{\nu}_{\mu}$ samples are divided into 28 and 19 even-sized energy bins in the interval [0.2, 3.0] GeV. The $\nu_{e}$ and $\bar{\nu}_{e}$ are collected from 23 equi-sized bins in $[0.1,1.25] \mathrm{GeV}$, while the $\nu_{e} \mathrm{CC} 1 \pi^{+}$sample has 16 bins in $[0.45,1.25] \mathrm{GeV}$.

\subsubsection{The NO $\nu$ A facility}

The $\mathrm{NO} \nu \mathrm{A}$ experiment [24] generates muon neutrino and antineutrino beams with the use of pion decay at $0.7 \mathrm{~kW}$ average power in the NuMI beam facility based in Fermilab in Illinois, U.S.A.. $\mathrm{NO} \nu \mathrm{A}$ has a near detector located at $1 \mathrm{~km}$ from the beam facility and a far detector facility at $810 \mathrm{~km}$ distance in Ash River, Minnesota. The $\mathrm{NO} \nu \mathrm{A}$ near and far detectors are totally active liquid scintillator detectors with about 193 ton and 14 kton fiducial masses. Both detectors are placed $0.8^{\circ}$ off-axis from the source. The neutrinos and antineutrinos produced in $\mathrm{NO} \nu \mathrm{A}$ spread over a wide range of energies around $2 \mathrm{GeV}$, and they may undergo a variety of charged-current interactions. The neutrinos and antineutrinos are detected through the scintillation light emitted by the charged particles created in the interaction.

For our work, we simulate the $\mathrm{NO} \nu \mathrm{A}$ data corresponding to $12.33 \times 10^{20}$ POT exposure in the NuMI source [24]. The data consists of five different samples for electron-like events and two samples for muon-like events. The four electron-like samples are categorized into two categories, which are defined by the purity of the event: low-PID and high-PID. ${ }^{5}$ The muon-like samples are split into 19 unequally spaced energy bins in the range [0.75, 4.0] GeV, whereas electron-like samples are separated into 6 bins in $[1.0,4.0] \mathrm{GeV}$. The fifth sample is the so-called peripheral sample. In this work, we consider the samples for the electron-like and muon-like events, while the peripheral sample is left out of the analysis.

\subsection{Reactor antineutrino experiments Daya Bay, Double Chooz and RENO}

Reactor antineutrino experiments are oscillation experiments where the emission of electron antineutrinos are observed from nuclear reactors. Reactor experiments are sensitive to $\bar{\nu}_{e} \rightarrow \bar{\nu}_{e}$ disappearance, which provide access to a set of dimension-6 SMEFT operators

\footnotetext{
${ }^{5}$ PID stands for particle identification. The high-PID and low-PID are categories for the convolutional neural network that categorizes neutrino events by their purity.
} 
different from what can be studied in the LBL experiments. We consider the presently running reactor neutrino experiments Daya Bay [25], Double Chooz [28] and RENO [30] in this work.

Daya Bay is a long-running reactor neutrino experiment based in southern Guangdong, China [25]. The Daya Bay experiment was the first to report simultaneous measurements of reactor antineutrinos at multiple baselines. The experiment led to the discovery of $\bar{\nu}_{e}$ oscillations over km-long baseline lengths. The experimental compound consists of six reactors and eight antineutrino detectors. Each of the nuclear reactors is estimated to have an output of about $2.9 \mathrm{GW}$ thermal power. The antineutrino detectors contain $20 \mathrm{t}$ of Gd-doped liquid scintillator in fiducial mass. The detectors are placed in pairs in three locations, in two near detector halls EH1 and EH2, and in a single far detector hall EH3. The detector compound is equivalent to $160 \mathrm{t}$ of liquid scintillator. Daya Bay has collected 1958 days of data since its launch in 2011, and it will continue to run until the end of 2020 . In the present work, we consider the oscillation data that corresponds to the 1958 days of data taking in Daya Bay [27].

Double Chooz is a short-baseline reactor neutrino experiment currently operating in France [28]. Double Chooz uses two identical Gd-doped liquid scintillators, which are both placed in the vicinity of two $4.2 \mathrm{GW}$ thermal power reactors. The near and far detectors are located approximately 400 and 1050 meters from the reactor cores, respectively. The experiment first started operation in 2005, when nuclear reactors were offline. This allowed the measurement of the backgrounds without the reactor flux. In this work, we consider antineutrino oscillation data corresponding to 1276 days of running with the Double Chooz far detector, and 587 days with the near detector, respectively. Both detectors are assumed to contain $10.6 \mathrm{t}$ of liquid scintillator. The results of the Double Chooz experiment were reported in ref. [29].

The third presently-running reactor neutrino experiment considered in this work is the RENO in South Korea [30]. RENO is based on the same working principle as Daya Bay and Double Chooz, and it has been taking data with two identical detectors from 2011. The two detectors are placed in near and far locations of $300 \mathrm{~m}$ and $1400 \mathrm{~m}$ from the reactor compound, and they host a total of $40 \mathrm{t}$ of liquid scintillator in fiducial mass. The experiment has observed the disappearance of reactor neutrinos in their interactions with free protons, followed by neutron capture on hydrogen $(\mathrm{n}-\mathrm{H})$. RENO has collected 2508 and 2908 days of data in its near and far detectors with $16.8 \mathrm{GW}$ thermal power [31].

In this work, we simulate the neutrino oscillation experiments with GLoBES, and successfully reproduce the results reported for $\mathrm{T} 2 \mathrm{~K}$ and $\mathrm{NO} \nu \mathrm{A}$ in refs. [21, 73] and ref. [24], as well as the results reported for Daya Bay, Double Chooz and RENO experiments in the Neutrino 2020 conference [71]. We then study constraints on dimension-6 SMEFT operators following the procedure shown in figure 1 and described in more detail in sections 2 and 3. The results are presented for a simplified scalar leptoquark model in section 5 and for the dimension-6 SMEFT operators in section 6 . Before going to the results directly, we first discuss the methods we use for the numerical analysis in the next subsection. 


\subsection{Numerical analysis}

The analysis presented in this work is performed with the GLoBES software $[35,36]$ for neutrino oscillation experiments. In order to compute the probabilities with NSIs, the add-on New Physics has been included [37].

In all considered experiments, the simulated neutrino oscillation data is analysed with $\chi^{2}$ functions, which span over energy bins $i=1,2, \ldots$ and detectors $d$. In the case of LBL experiments, the $\chi^{2}$ function used in the analysis is

$$
\chi^{2}=\sum_{d}\left(\sum_{i} 2\left[T_{i, d}-O_{i, d}\left(1+\log \frac{O_{i, d}}{T_{i, d}}\right)\right]+\frac{\zeta_{\mathrm{sg}}^{2}}{\sigma_{\zeta_{\mathrm{sg}}}^{2}}+\frac{\zeta_{\mathrm{bg}}^{2}}{\sigma_{\zeta_{\mathrm{bg}}}^{2}}\right)+\text { priors },
$$

where $O_{i, d}$ and $T_{i, d}$ stand for the observed and theoretical/predicted events in the near and the far detectors, which are denoted with $d=N$ and $F$, respectively. The systematic uncertainties are addressed with the so-called pull-method [74]. We consider normalized errors for signal and background events with nuisance parameters $\zeta_{\mathrm{sg}}$ and $\zeta_{\mathrm{bg}}$, which influence the predicted events $T_{i, d}$ in near and far detectors with a simple shift: $T_{i, d}=\left(1+\zeta_{\mathrm{sg}}\right) N_{i, d}^{\mathrm{sg}}+\left(1+\zeta_{\mathrm{bg}}\right) N_{i, d}^{\mathrm{bg}}$ where $N_{i, d}^{\mathrm{sg}}$ and $N_{i, d}^{\mathrm{bg}}$ are signal and background events, respectively. The prior function is defined as the Gaussian distributions of each of the standard neutrino oscillation parameters as well as the non-standard interaction parameters. We adopt the central values and associated errors from the present fit on the world data.

In the case of the reactor neutrino experiments, it is sufficient to use the $\chi^{2}$ function

$$
\begin{aligned}
\chi^{2}= & \sum_{d} \sum_{i} \frac{\left(O_{d, i}-T_{d, i}\left(1+a_{\mathrm{norm}}+\zeta_{d}+\beta_{d}+\xi_{d}\right)\right)^{2}}{O_{d, i}} \\
& +\sum_{d}\left(\frac{\zeta_{d}^{2}}{\sigma_{\zeta}^{2}}+\frac{\xi_{d}^{2}}{\sigma_{\xi}^{2}}+\frac{\beta_{d}^{2}}{\sigma_{\beta}^{2}}\right)+\frac{a_{\text {norm }}^{2}}{\sigma_{a}^{2}}+\text { priors }
\end{aligned}
$$

where $d$ runs through all detectors in the reactor experiment. The observed and theoretical events are given for detector $d$ and energy bin $i$ by $O_{d, i}$ and $T_{d, i}$, respectively. The nuisance parameters $\zeta_{d}, \xi_{d}$ and $\beta_{d}$ and their Gaussian widths are assigned to address the systematic uncertainties regarding the efficiency, energy calibration and scaling in the detectors, respectively. The nuisance parameter $a_{\text {norm }}$ is related to the overall normalization error in the reactor rates.

As it is apparent from the $\chi^{2}$ functions used for accelerator and reactor experiments in eqs. (4.1)-(4.2), the statistical analysis is based on the log-likelihood and likelihood tests, respectively. The $\chi^{2}$ function for LBL experiments shown in eqs. (4.1) follows Poissonian statistics and is suitable for experiments with relatively low statistics, such as T2K and $\mathrm{NO} \nu \mathrm{A}$. For reactor experiments Daya Bay, Double Chooz and RENO on the other hand, the Gaussian distribution used in eq. (4.2) is suitable for the statistical analysis.

The details of the considered LBL experiments can be found in table 2. The reactor experiments are summarized in table 1. The near and the far units of the Daya Bay experiment refer to the detector compounds in EH1 and EH2, and EH3, respectively. The simulated data is computed from the present best-fit values of the neutrino oscillation parameters from the world data, summarized in table 3. We adopt priors for the standard oscillation parameters from the best-fit values shown in the table. 


\begin{tabular}{|cccc|}
\hline Experiment & Double Chooz & Daya Bay & RENO \\
\hline Location & France & China & South Korea \\
Thermal power & $8.4 \mathrm{GW}$ & $17.4 \mathrm{GW}$ & $16.4 \mathrm{GW}$ \\
Exposure (far) & 1276 days & 1985 days & 2908 days \\
Exposure (near) & 587 days & 1985 days & 2509 days \\
Fiducial mass (far) & $10.6 \mathrm{t}$ & $80 \mathrm{t}$ & $15.4 \mathrm{t}$ \\
Fiducial mass (near) & $10.6 \mathrm{t}$ & $2 \times 40 \mathrm{t}$ & $15.4 \mathrm{t}$ \\
Baseline length (far) & $1.05 \mathrm{~km}$ & $1.579 \mathrm{~km}$ & $1.4 \mathrm{~km}$ \\
Baseline length (near) & $400 \mathrm{~m}$ & $512 \mathrm{~m}, 561 \mathrm{~m}$ & $300 \mathrm{~m}$ \\
\hline References & Ref. $[28]$ & Refs. $[25,26]$ & Ref. $[30]$ \\
\hline
\end{tabular}

Table 1. Reactor neutrino experiments considered in this study. Each of the reactor experiments have two or more detectors based in different locations. The baseline lengths correspond to the average distance between the reactors and detector compounds.

\begin{tabular}{|ccc|}
\hline Experiment & T2K & NO $\nu \mathrm{A}$ \\
\hline Source location & Japan & U.S.A. \\
operating & operating \\
Status & $770 \mathrm{~kW}$ & $700 \mathrm{~kW}$ \\
Beam power & $1.3 \times 10^{21}$ & $12.33 \times 10^{20}$ \\
Protons-on-target & $22.5 \mathrm{kt}$ & $14 \mathrm{kt}$ \\
Fiducial mass (far) & $1.529 \mathrm{t}$ & $193 \mathrm{t}$ \\
Fiducial mass (near) & $295 \mathrm{~km}$ & $810 \mathrm{~km}$ \\
Baseline length (far) & $1 \mathrm{~km}$ \\
Baseline length (near) & $280 \mathrm{~m}$ & $0.8^{\circ}$ \\
Off-axis angle & $2.5^{\circ}$ & Ref. $[24]$ \\
\hline References & Ref. $[21]$ & \\
\hline
\end{tabular}

Table 2. The long-baseline neutrino experiments simulated in this study.

\begin{tabular}{|ccc|}
\hline Parameter & Central value $\pm 1 \sigma(\mathrm{NO})$ & Central value $\pm 1 \sigma(\mathrm{IO})$ \\
\hline$\theta_{12}\left(^{\circ}\right)$ & $33.440 \pm 0.755$ & $33.450 \pm 0.765$ \\
$\theta_{13}\left(^{\circ}\right)$ & $8.570 \pm 0.120$ & $8.600 \pm 0.120$ \\
$\theta_{23}\left(^{\circ}\right)$ & $49.200 \pm 1.050$ & $49.300 \pm 1.000$ \\
$\delta_{\mathrm{CP}}\left(^{\circ}\right)$ & $197.000 \pm 25.500$ & $282.000 \pm 28.000$ \\
$\Delta m_{21}^{2}\left(10^{-5} \mathrm{eV}^{2}\right)$ & $7.420 \pm 0.205$ & $7.420 \pm 0.205$ \\
$\Delta m_{3 l}^{2}\left(10^{-3} \mathrm{eV}^{2}\right)$ & $2.517 \pm 0.027$ & $-2.498 \pm 0.028$ \\
\hline
\end{tabular}

Table 3. The best-fit values and $1 \sigma$ confidence level (CL) uncertainties in the standard threeneutrino mixing $[3,75]$. The values are shown for both normal ordering (NO) and inverted ordering (IO), where $\Delta m_{3 \ell}^{2}$ corresponds to $\Delta m_{31}^{2}(\mathrm{NO})$ and $\Delta m_{32}^{2}$ (IO), respectively. 
The prior values we choose to use in this work are based on the three-neutrino oscillation fit assuming no non-standard interactions. As the numerical analysis we show in this work is about assessing the neutrino NSI that has origin in the new physics occurring at the high scale, the extended framework should in principle be accounted in the priors as well. As we shall see, however, the impact of such extension on our numerical results falls below the precision on our work.

\subsection{Constraining NSI parameters in neutrino experiments}

We illustrate the power of using the neutrino oscillation experiments in constraining new physics with the following example. By taking only one source or detection NSI parameter $\epsilon_{\alpha \beta}^{s / d}(\alpha, \beta=e, \mu, \tau)$ to be non-zero at a time, we obtain the constraints on the magnitude of the NSI parameters. Since the nature of the source and detection NSI depends on the specific neutrino production and detection method used in the experiment, we simulate the LBL and reactor experiments separately. Since no significant deviations from the standard three-neutrino oscillations has been found in the considered experiments, we obtain the upper bounds for each NSI parameter with the following method.

The $\chi^{2}$ distribution is computed as $\Delta \chi^{2} \equiv \chi_{\mathrm{NSI}}^{2}-\chi_{\mathrm{SI}}^{2}$, where $\chi_{\mathrm{NSI}}^{2}$ is computed with the appropriate $\chi^{2}$ function assuming one NSI parameter to be non-zero and $\chi_{\text {SI }}^{2}$ with all NSI parameters zero. The resulting $\Delta \chi^{2}$ distribution follows approximately a $\chi^{2}$ distribution of one degree of freedom. The $\chi_{\mathrm{NSI}}^{2}$ and $\chi_{\mathrm{SI}}^{2}$ are minimized over the standard neutrino oscillation parameters with related priors. The sensitivity to each NSI parameter is then projected at $95 \%$ CL of statistical significance by requiring $\Delta \chi^{2}=3.8416$.

We present the upper bounds on the source and detection NSI parameters for the LBL and reactor experiments in table 4 . The results are obtained for the absolute values of the NSI parameters at $95 \% \mathrm{CL}$. We consider only the source NSI parameters $\epsilon_{\mu e}^{s}, \epsilon_{\mu \mu}^{s}$ and $\epsilon_{\mu \tau}^{s}$ for the LBL experiments, while the source and detection parameters $\epsilon_{e \beta}^{s}$ and $\epsilon_{\alpha e}^{d}(\alpha$, $\beta=e, \mu, \tau)$ are studied in the reactor experiments. As one shall see from the table, the experiments provide the most stringent results on $\left|\epsilon_{\mu e}^{s}\right|$ in pion decay, $\left|\epsilon_{e e}^{s}\right|$ in beta decay and $\left|\epsilon_{e e}^{d}\right|$ in inverse beta decay. ${ }^{6}$

The upper bounds on the source and detection NSI parameters presented here are specified for the case where only one NSI parameter bears a non-zero value. If one should consider a scenario where more than one NSI parameter has a non-negligible value, the constraints on the individual NSI parameters are relaxed. Furthermore, any of these NSI parameters may also have a complex phase. Hence, the results presented in table 4 describe the most optimistic setup. In the following sections of this work, it is assumed the magnitude and phase of the NSI parameters can be calculated precisely.

The presence of the neutrino NSI could also affect the prior values applicable for this work, as noted in the previous section. The effect of the NC NSI, for example, can lead to the emergence of the LMA-D scenario, where the precision to the solar parameters $\theta_{12}$ and $\Delta m_{21}^{2}$ is affected by the appearing of a local minimum [76]. We investigated the effect

\footnotetext{
${ }^{6}$ We note that the results presented in table 4 are sensitive to how the experimental setups are described in the definition language in GLoBES. Whereas the non-oscillation backgrounds played a crucial part in defining the sensitivity to the reactor neutrino experiments, we found the near detector to be particularly important in the long-baseline neutrino experiments.
} 


\begin{tabular}{|rrl|}
\hline NSI parameter & Upper bound & Experiments \\
\hline$\left|\epsilon_{\mu e}^{s}\right|$ & 0.004 & \\
$\left|\epsilon_{\mu \mu}^{s}\right|$ & 0.021 & T2K $[21,72,73]$, NO $\nu \mathrm{A}[24]$ \\
$\left|\epsilon_{\mu \tau}^{s}\right|$ & 0.080 & \\
\hline$\left|\epsilon_{e e}^{d}\right|$ & 0.007 & \\
$\left|\epsilon_{\mu e}^{d}\right|$ & 0.018 & \\
$\left|\epsilon_{\tau e}^{d}\right|$ & 0.021 & Daya Bay $[25,27]$, Double Chooz $[28,29]$, \\
$\left|\epsilon_{e e}^{s}\right|$ & 0.007 & and RENO [30,31] \\
$\left|\epsilon_{e \mu}^{s}\right|$ & 0.018 & \\
$\left|\epsilon_{e \tau}^{s}\right|$ & 0.021 & \\
\hline
\end{tabular}

Table 4. Upper bounds of the NSI parameters associated with the neutrino production and detection. All constraints are given at 95\% CL.

of the relaxed solar parameter constraints in the NSI sensitivities presented in table 4 , however, we found no significant change. ${ }^{7}$

\section{A UV example: the simplified scalar leptoquark model}

To illustrate how the matching and the running work, as well as how the numerical analysis of neutrino oscillation experiments discussed in last section can be used to impose constraints on the UV models, we adopt the simplified scalar leptoquark model in this section. We will first set up this simple UV model and then illustrate how neutrino experiments are used to constrain this model. As shown in figure 1, since this simplified model is defined at a UV scale much above that at which neutrino experiments are carried out, the running effects need to be considered. The Wilson package is used for the running and the matching at the weak scale, after which a comparison between theoretical prediction from this simplified UV model and experimental bounds on the NSI parameters is done to obtain constraints on this model. As we will see later in this section, though neutrino experiments are performed at a very low energy scale, they put much more stringent constraints than those from high-energy experiments at colliders.

\subsection{Model setup and connection to neutrino oscillation experiments}

Our simplified leptoquark model only contains a scalar leptoquark $S$ with the SM quantum number $(\overline{3}, 1,1 / 3)$, the renormalizable Lagrangian is given by [77]

$$
\begin{aligned}
\mathcal{L}_{\mathrm{LQ}}= & \left|D_{\mu} S\right|^{2}-M_{1}^{2}|S|^{2}-\lambda_{H 1}|H|^{2}|S|^{2}-\frac{c}{2}|S|^{4} \\
& +\left(\left(\lambda^{L}\right)_{i \alpha} \bar{q}_{i}^{c} \epsilon \ell_{\alpha}+\left(\lambda^{R}\right)_{i \alpha} \bar{u}_{i}^{c} e_{\alpha}\right) S_{1}+\text { h.c. }
\end{aligned}
$$

\footnotetext{
${ }^{7}$ The neutrino NSI discussed in ref. [76] is in fact of different nature compared to the NSI we investigate in the present work. Whereas the former work focuses on the NC NSI originating from light mediators, the CC NSI we investigate in this work has origin at the UV scale.
} 
with $\epsilon=i \sigma^{2}$ and $\sigma^{2}$ the second Pauli matrix. In principle, there also exist baryon number violation terms $\bar{q}^{c} \epsilon q S^{*}$ and $\bar{u}^{c} d S^{*}$, but they are extremely suppressed due to the constraint from proton decay, we thus simply ignore them in our setup. Integrating out the heavy leptoquark $S$, one finds this model contributes to the following dimension-6 SMEFT operators in the Warsaw-down basis at tree level [77]: ${ }^{8}$

$$
\begin{aligned}
& \mathcal{O}_{\ell q}^{(1)} \equiv\left(\bar{\ell} \gamma^{\mu} \ell\right)\left(\bar{q} \gamma_{\mu} q\right), \quad \mathcal{O}_{\ell q}^{(3)} \equiv\left(\bar{\ell} \gamma^{\mu} \sigma^{I} \ell\right)\left(\bar{q} \gamma_{\mu} \sigma^{I} q\right), \quad \mathcal{O}_{\ell e q u}^{(1)} \equiv\left(\bar{\ell}^{r} e\right) \epsilon_{r s}\left(\bar{q}^{s} u\right), \\
& \mathcal{O}_{\ell e q u}^{(3)} \equiv\left(\bar{\ell}^{r} \sigma^{\mu \nu} e\right) \epsilon_{r s}\left(\bar{q}^{s} \sigma_{\mu \nu} u\right), \quad \mathcal{O}_{e u} \equiv\left(\bar{e} \gamma^{\mu} e\right)\left(\bar{u} \gamma_{\mu} u\right)
\end{aligned}
$$

whose Wilson coefficients are given by, respectively [77], ${ }^{9}$

$$
\begin{aligned}
& \underset{\alpha \beta i j}{C_{l q}^{(1)}}=\frac{\lambda_{i \alpha}^{L *} \lambda_{j \beta}^{L}}{4 M^{2}}, \quad C_{\alpha \beta i j}^{(3)}=-\frac{\lambda_{i \alpha}^{L *} \lambda_{j \beta}^{L}}{4 M^{2}}, \quad C_{l e q u}^{(1)}=\frac{\lambda_{j \beta}^{R} \lambda_{i \alpha}^{L *}}{2 M^{2}}, \\
& C_{\text {lequ }}^{(3)}=-\frac{\lambda_{j \beta}^{R} \lambda_{i \alpha}^{L *}}{8 M^{2}}, \quad C_{\alpha \beta i j}^{e u}=\frac{\lambda_{i \alpha}^{R *} \lambda_{j \beta}^{R}}{2 M^{2}},
\end{aligned}
$$

where $r, s$, denote the fundamental representations of the $\mathrm{SU}(2)_{\mathrm{L}}$ group, $\alpha, \beta$ stand for lepton flavor indices, and $i, j$ represent quark flavors.

Matching this simplified scalar leptoquark model onto the SMEFT is exactly the first step in the first row of figure 1, and the Wilson coefficients after this matching are all defined at $\mu=M$ with $M$ here being the UV scale $\Lambda$. To connect this UV model to the low-energy neutrino experiments represented by the last row of figure 1, the Wilson package is utilized. To be more specific for the purpose of illustrating our approach, we discuss the intermediate steps next.

We first take the dimension-6 SMEFT operators obtained from this simplified scalar leptoquark model and defined in the Warsaw-down basis in eq. (5.2)-(5.3) as the input for the Wilson package. Simultaneously, we set all the other dimension-6 SMEFT operators to zero at the input scale $\mu=M$ since we are interested in the prediction specifically from this simplified model. Next, based on the work in refs. [68-70], the Wilson package runs all the dimension-6 SMEFT operators down to the weak scale $m_{W}$ as indicated by the arrow connecting the purple and the green in figure 1. At this step, the SMEFT operators mix with each other during the running as a result of the entangled RGEs [67]. Therefore, vanishing SMEFT operators at the input scale do not necessarily imply that they lead to vanishing contributions at a different scale.

After the RGE running above, the Wilson package can also automatically match the SMEFT to the LEFT at $\mu=m_{W}$ based on refs. [62, 78], as well as a following RGE running from the weak scale $m_{W}$ down to the $2 \mathrm{GeV}$ scale for the LEFT based on the theoretical work of ref. [59]. These two intermediate steps are represented by the second row and the arrow connecting the green and the yellow in figure 1 respectively. The LEFT

\footnotetext{
${ }^{8}$ Contributions at one-loop level are also studied in ref. [77], but since their contributions to the Wilson coefficients during the RGE running are of two-loop order [66], and can thus be safely ignored. Therefore, we only focus on the tree-level results in this subsection for illustration.

${ }^{9}$ Note that different from the usual definition for Wilson coefficients, the UV scale $\Lambda=M$ is included here to reflect the dependence of the NSI parameters on both the couplings and the leptoquark mass.
} 
obtained at this scale serves as an ideal framework for the description of neutrino oscillation experiments, and the event rates $R_{\alpha \beta}$ can be expressed in terms of the Wilson coefficients $\epsilon_{L, R, S, P, T}$ in the LEFT in the QFT formalism. On the other hand, neutrino observables used to be expressed in terms of the source and detection NSI parameters $\epsilon^{s, d}$ in the QM formalism, and neutrino experimental results are presented as constraints on these $\epsilon^{s, d}$ parameters. Therefore, to connect the UV model directly to neutrino experiments, this last gap needs to be solved. A consistent matching between the QFT and the QM formalisms has recently been discussed in ref. [20] and is depicted by the last row of figure 1 .

Following the procedure described above, we present our study on this simplified scalar leptoquark model in the following subsections.

\subsection{Constraints on $M$ with $\lambda_{L, R}=1$}

As discussed in last subsection, newly introduced interactions between the leptoquark and SM particles in eq. (5.1) generate the tree-level dimension-6 SMEFT operators at the UV scale in eq. (5.2)-(5.3). As a consequence, they contribute to the charged- and neutralcurrent NSI parameters defined in eq. (2.3) at the $2 \mathrm{GeV}$ scale. Given the current very stringent constraints on these NSI parameters, the low-energy neutrino experiments can thus be applied to explore this simplified model. To that end, we first use the GLoBES package to obtain the constraints on each source and detection NSI parameter, summarized in table 4. We then use the Wilson package to find the prediction on the same set of NSI parameters listed in table 4 from this UV model. We also perform this procedure in inverted order, where the output of Wilson is transferred to GLoBES to directly compute the exclusion limits to the leptoquark model.

In the Warsaw-down basis, since nearly 300 non-vanishing dimension-6 SMEFT operators contribute at the input scale $\Lambda$, for simplicity, we assume $\lambda_{L, R}$ are both real and then present our results in the top-left, top-right and bottom-left panels of figure 3. To obtain the plots, we fix $\lambda_{L, R}=1$ and focus on only one NSI parameter at a time. We use a horizontal dashed red line in each subfigure to represent the current upper bound on each NSI parameter summarized in table 4 . We conclude that, among all the NSI parameters listed in table $4,\left|\epsilon_{\mu e}^{s}\right|$ from pion decay leads to the most stringent constraint on the leptoquark model, and it presently excludes a leptoquark lighter than about $16 \mathrm{TeV}$ as indicated by the upper left plot in figure 3. Interestingly, we note that there is a tiny window around $M \in[2180,2310] \mathrm{GeV}$ that survives from current constraint on $\left|\epsilon_{\mu e}^{s}\right|$, and in general, this tiny region survives even if the experimental sensitivity on $\left|\epsilon_{\mu e}^{s}\right|$ is improved. However, if one takes other NSI parameters into account as shown in the upper right and/or lower left plots of figure 3, this tiny window would have already been excluded.

Another interesting point from figure 3 is that, though the current upper bound on $\left|\epsilon_{\mu \tau}^{s}\right|$ only excludes a leptoquark lighter than about $4 \mathrm{TeV}$, weakest among the three NSI parameters shown, this exclusion limit is still much stronger than that from collider studies at the ATLAS and the CMS [79, 80], implying the complementarity of high- and lowenergy experiments in searching for new physics. A similar observation regarding the complementarity has been obtained for many other low-energy precision experiments like the electric dipole moments in the leptoquark model, see ref. [81] for example. 

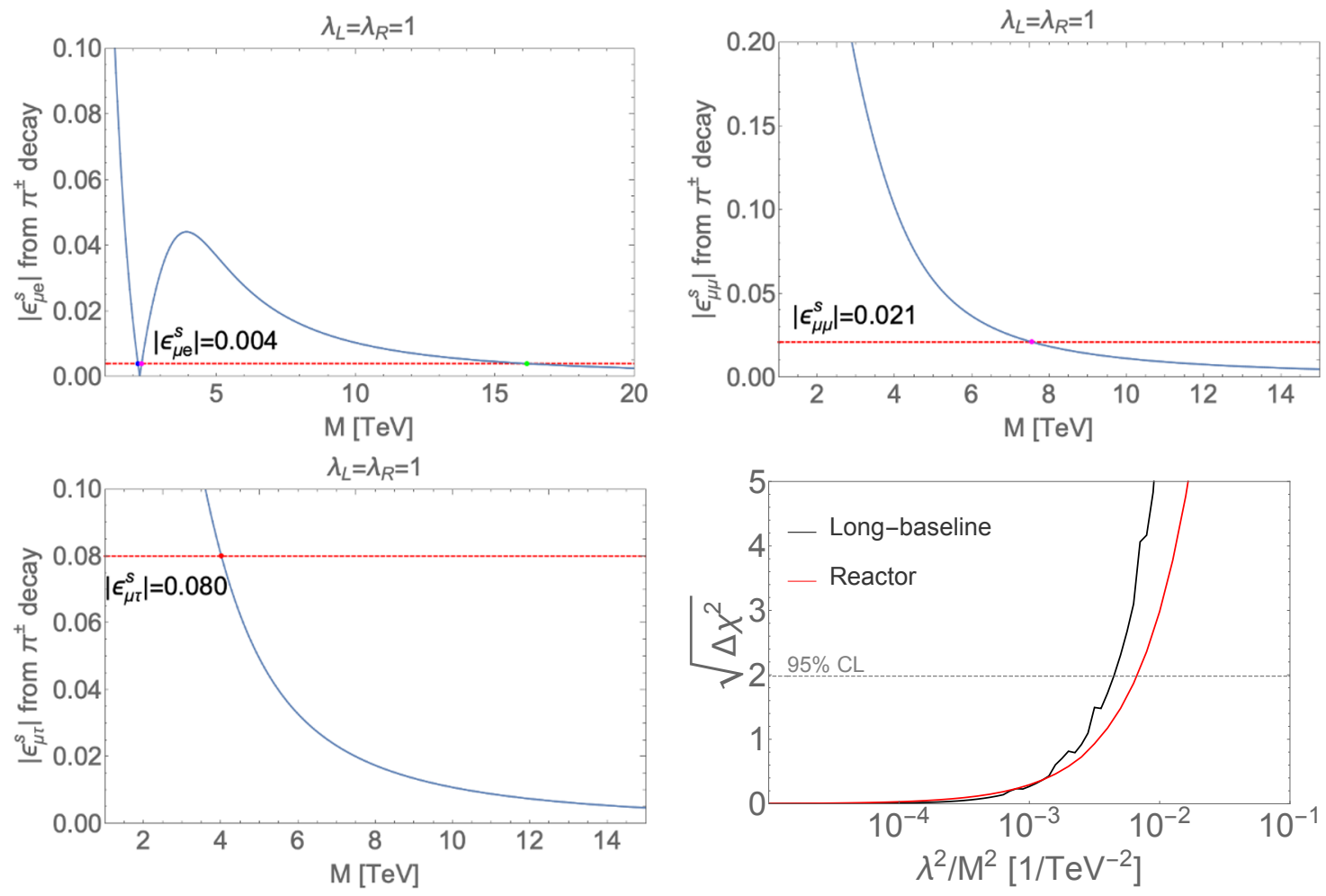

Figure 3. Constraints on the leptoquark mass from $\left|\epsilon_{\mu \alpha}^{s}\right|(\alpha=e, \mu, \tau)$ from pion decay. The horizontal red dashed line in each plot corresponds to the upper bounds on the selected NSI parameters summarized in table 4. Constraints on the NSI parameters from the beta and the inverse beta decay presently have no constraints on this model as can be seen in section 5.2.

Upper bounds on the other NSI parameters from the beta and the inverse beta decay turn out to impose no constraints on this simplified leptoquark model for $\lambda_{L, R}=1$, as one can see from figure 4.

We conclude our investigation with the lower-right panel of figure 3, where the overall sensitivity to the leptoquark model is shown both in the LBL experiments $\mathrm{T} 2 \mathrm{~K}$ and $\mathrm{NO} \nu \mathrm{A}$, and the reactor experiments Daya Bay, Double Chooz and RENO. The two sensitivity plots show the statistical significance at which the model can be excluded in the experiments as function of $\lambda^{2} / M^{2}$, whereby $\lambda \equiv \lambda_{L}=\lambda_{R}=1$ is assumed for simplicity. The $95 \%$ CL is showcased with the horizontal dashed line. At this statistical limit, the LBL experiments perform better at $\lambda^{2} / M^{2} \lesssim 4.4 \times 10^{-3} 1 / \mathrm{TeV}^{2}$, whereas the reactor experiment set the bound at $\lambda^{2} / M^{2} \lesssim 6.5 \times 10^{-3} 1 / \mathrm{TeV}^{2}$. The corresponding masses are approximately $15 \mathrm{TeV}$ and $12.5 \mathrm{TeV}$ respectively. The results from the LBL and reactor experiments are therefore comparable, which are also stronger than the constraints from ATLAS and CMS.

\subsection{Constraints on $\lambda_{L, R}$ with fixed $M$}

On the other hand, since $\lambda_{L, R}$ are particularly interesting for collider studies, we also present our results with fixed leptoquark masses in figure 5 upon scanning over $\lambda_{L, R}$. The 

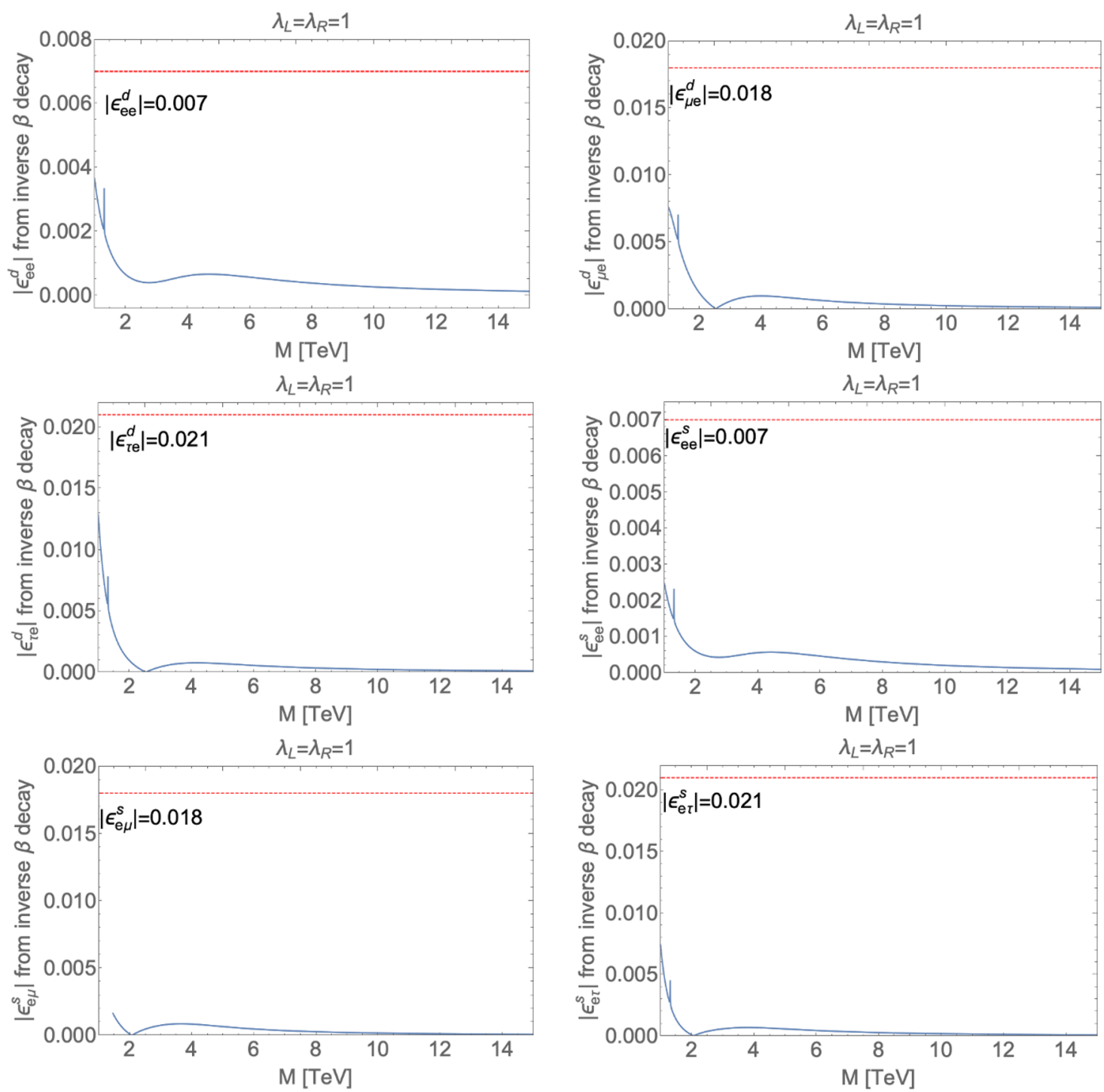

Figure 4. Constraints on the simplified scalar leptoquark model from the neutrino NSI parameters from beta decay and inverse beta decay. The horizontal red dashed line in each plot corresponds to the current upper bound on the NSI parameter, and the blue curve corresponds to theoretical prediction from this simplified model.

pink region(s) in each plot of figure 5 is (are) still allowed at $95 \% \mathrm{CL}$, obtained from a comparison between theoretical prediction from this simplified model and the experimental upper bounds on the neutrino NSI parameters summarized in table 4.

Similar to what we observe in section 5.2, the current constraint on $\left|\epsilon_{\mu e}^{s}\right|$ from pion decay dominates all the other NSI parameters in table 4, which can be seem from the first column of figure 5. Particularly, in the case where $M=1 \mathrm{TeV}$, the current constraint on $\left|\epsilon_{\mu e}^{s}\right|$ excludes almost the entire parameter space of this simplified model unless $\lambda_{L}$ and/or $\lambda_{R}$ are very tiny to suppress contributions from the UV physics. This point will become clearer if one matches the simplified UV model to the LEFT directly at the UV scale $\mu=M$ 

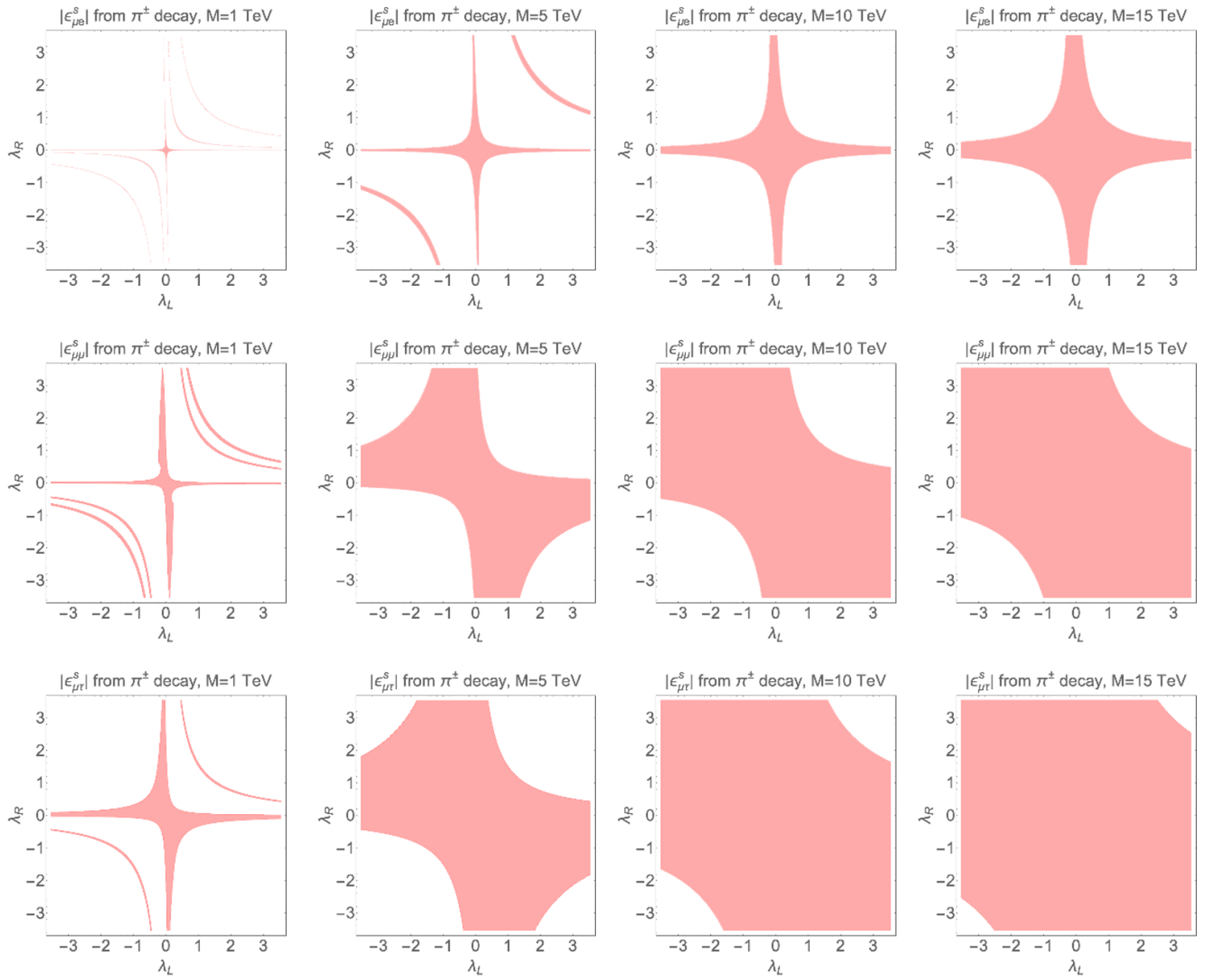

Figure 5. Constraints on the simplified leptoquark model parameter space from current constraints on $\left|\epsilon_{\mu \ell}^{s}\right|(\ell=e, \mu, \tau)$ summarized in table 4. The pink region(s) in each figure is (are) still allowed at $95 \%$ CL, obtained by fixing $\lambda_{L}=\lambda_{R}=1$. See the main text for details.

without taking into account any subleading running effects, which gives ${ }^{10}$

$$
\begin{aligned}
& \epsilon_{\mu \beta}^{s}(\mu=M)=\left(1+\frac{m_{\pi}^{2}}{m_{\mu}\left(m_{u}+m_{d}\right)}\right) \frac{v^{2} \lambda_{L} \lambda_{R}}{4 M^{2}}\left(\frac{\sum_{x=d, s, b} V_{u x}^{*}}{V_{u d}}\right)^{*}, \\
& \epsilon_{e \beta}^{s}(\mu=M)=\left(1+\frac{g_{T}}{g_{A}} \frac{m_{e}}{f_{T}\left(E_{\nu}\right)}\right) \frac{v^{2} \lambda_{L} \lambda_{R}}{4 M^{2}}\left(\frac{\sum_{x=d, s, b} V_{u x}^{*}}{V_{u d}}\right)^{*}, \\
& \epsilon_{\beta e}^{d}(\mu=M)=\left(1+\frac{m_{e}}{E_{\nu}-\Delta} \frac{g_{S}-3 g_{A} g_{T}}{1+3 g_{A}^{2}}\right) \frac{v^{2} \lambda_{L} \lambda_{R}}{4 M^{2}}\left(\frac{\sum_{x=d, s, b} V_{u x}^{*}}{V_{u d}}\right)^{*} .
\end{aligned}
$$

Clearly, when $\lambda_{L}$ and/or $\lambda_{R}$ are tiny, contributions to the NSI parameters from this simplified model are suppressed for any fixed $M$. Alternatively, equivalent suppression can

\footnotetext{
${ }^{10}$ Note that we assume both $\lambda_{L}$ and $\lambda_{R}$ are real for simplicity.
} 

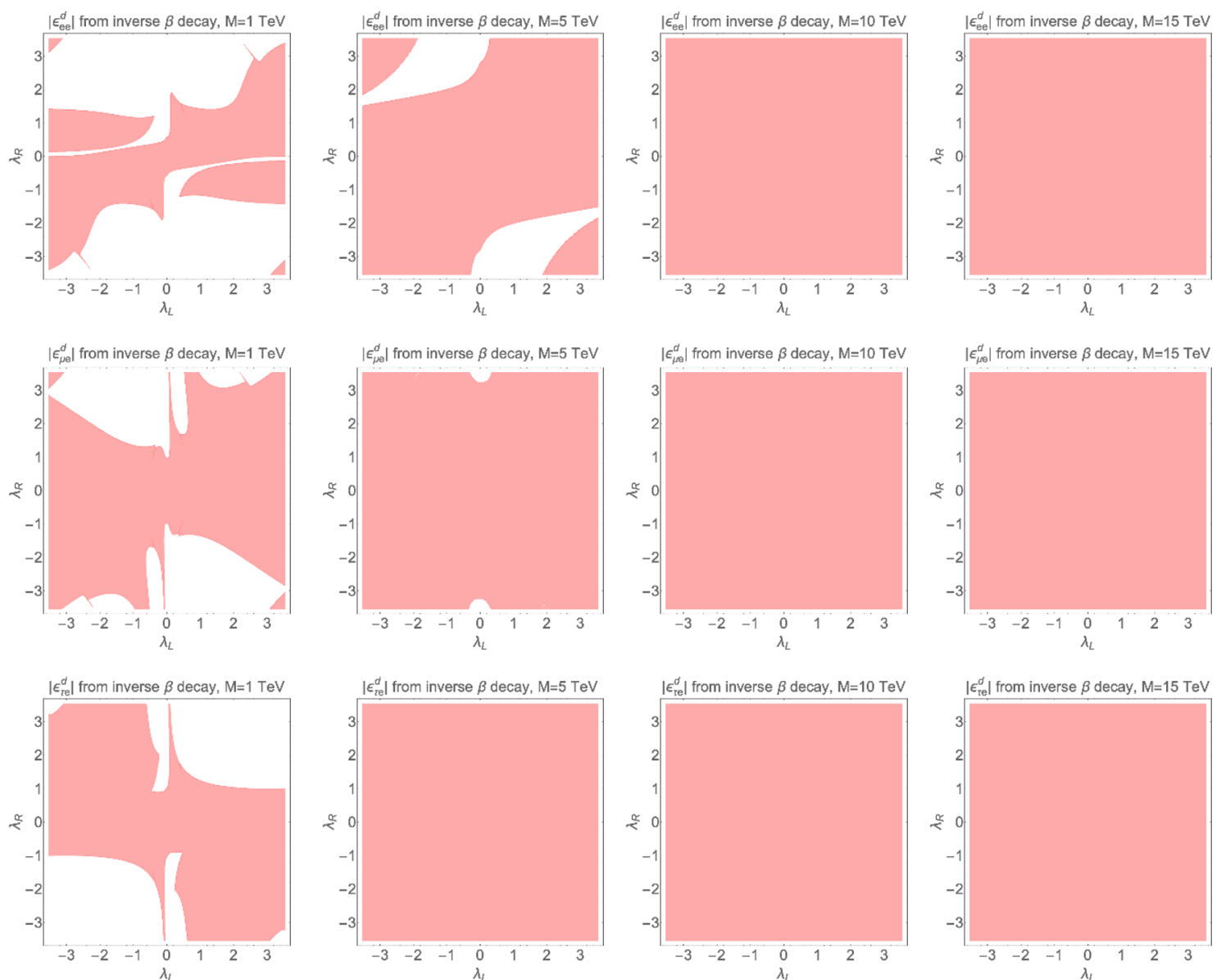

Figure 6. Constraints on the simplified leptoquark model parameter space from current constraints on $\left|\epsilon_{\ell e}^{d}\right|(\ell=e, \mu, \tau)$ from inverse beta decay summarized in table 4 . The pink region(s) in each figure is (are) still allowed at 95\% CL, obtained with fixed leptoquark mass $M$.

be achieved by increasing $M$, as a result, moderate or even large $\lambda_{L, R}$ are allowed as can be seen from the last three columns of figure 5. However, we point out that, even when the leptoquark mass is large of $\mathcal{O}(10 \mathrm{TeV}), \lambda_{L}$ and $\lambda_{R}$ can not simultaneously become large to survive from the current constraint on $\left|\epsilon_{\mu e}^{s}\right|$, shown in the last plot in the first row of figure 5. Constraints from other NSI parameters turn out to be relatively weaker than those shown in figure 5, as one can see clearly from figures 6 and 7 .

\section{Constraints on the dimension-6 SMEFT operators from neutrino os- cillation experiments}

In the last section, the robustness of using neutrino NSI parameters to study new physics at high energies is illustrated by the simplified scalar leptoquark model. In this section, following figure 1, we generalize our study to all dimension-6 SMEFT operators and present our final results as lower constraints on the UV scale $\Lambda$ as well as upper constraints on the Wilson coefficients pertaining to individual operators. Due to the large number of 

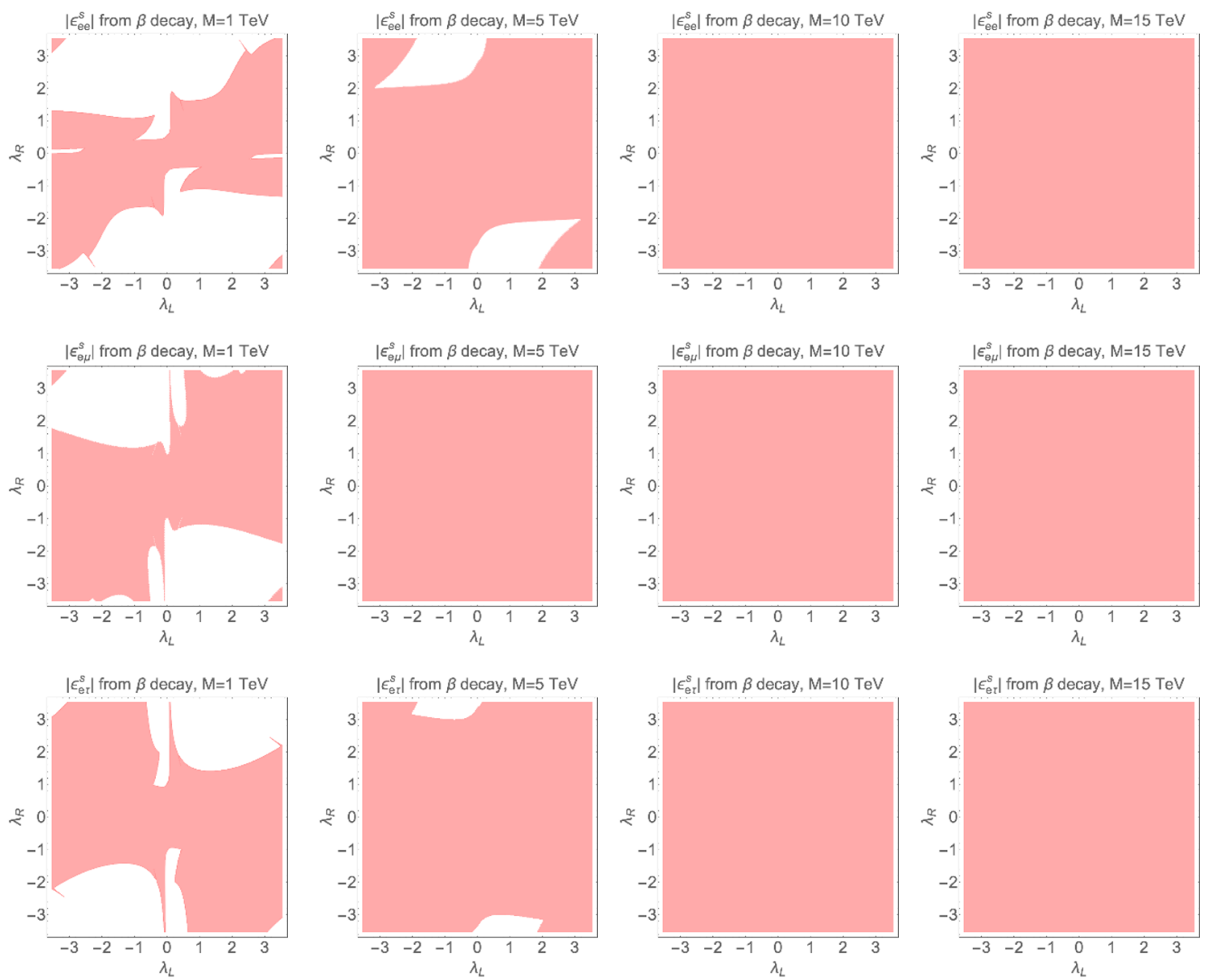

Figure 7. Constraints on the simplified leptoquark model parameter space from current constraints on $\left|\epsilon_{e \ell}^{s}\right|(\ell=e, \mu, \tau)$ from beta decay summarized in table 4. The pink region(s) in each figure is (are) still allowed at $95 \% \mathrm{CL}$, obtained with fixed leptoquark mass $M$.

dimension-6 SMEFT operators, we only present the results for operators that are within the reach of the neutrino experiments considered in this work. To that end, we first take the bottom-up EFT approach where one assumes all the dimension-6 SMEFT operators are independent at the UV scale $\Lambda$ and then derives the experimental constraints on $\Lambda$ and the Wilson coefficient of each operator. The results are presented in subsection 6.1 and achieved by simulating the neutrino oscillation data in the LBL neutrino experiments $\mathrm{T} 2 \mathrm{~K}$ and $\mathrm{NO} \nu \mathrm{A}$ and the reactor antineutrino experiments Daya Bay, Double Chooz and RENO.

On the other hand, as discussed in section 3, the correlation among different operators will in general get lost in the bottom-up EFT approach. However, as already can be seen in the simplified scalar leptoquark model case presented in section 5 , effects due to the correlation among the dimension-6 SMEFT operators already show up at the UV scale $\Lambda$. As a result, neutrino NSI parameters at the $2 \mathrm{GeV}$ scale will also be affected by the correlation. We present our results demonstrating the correlation among different operators in subsection 6.2 . 

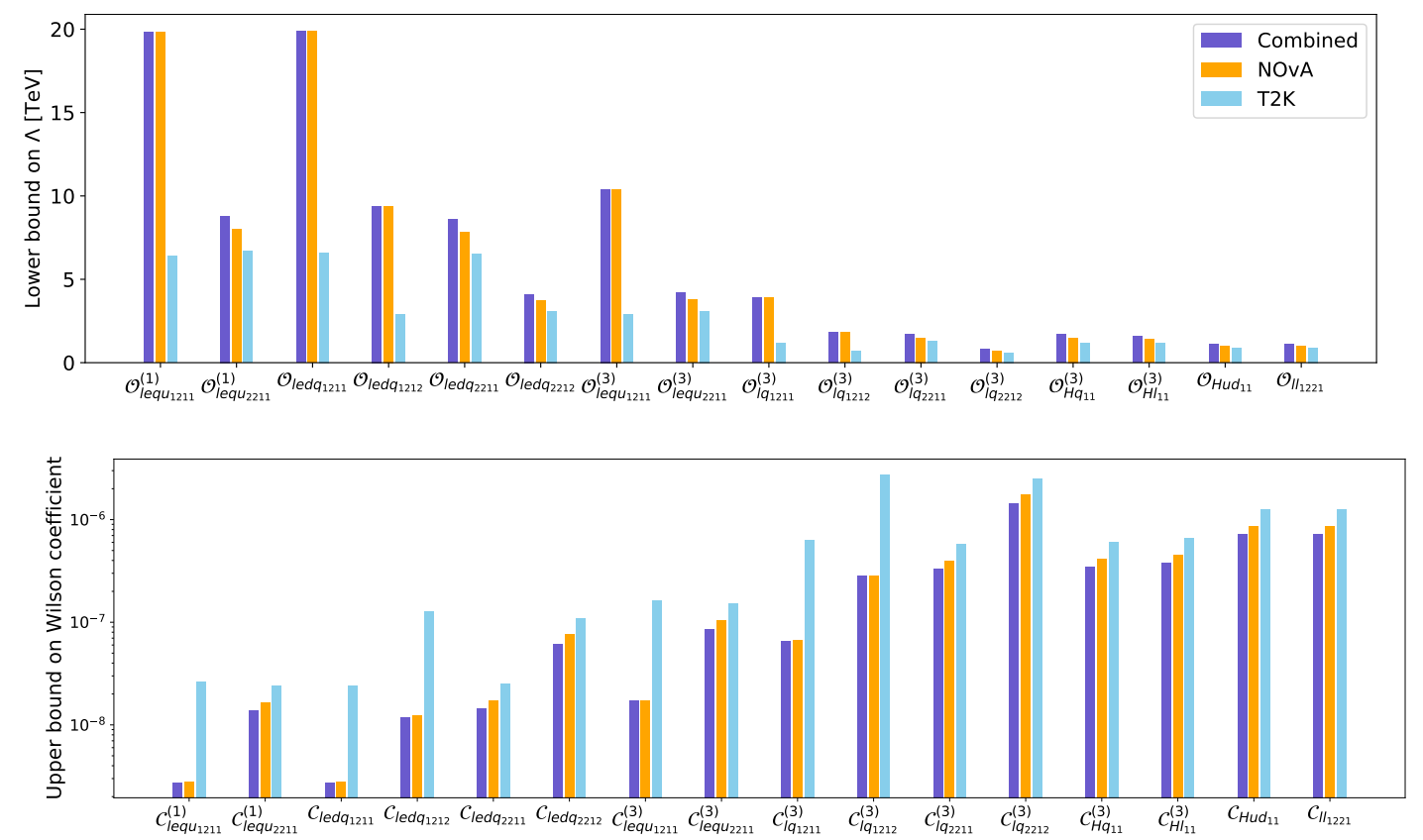

Figure 8. Constraints on a part of dimension- 6 operators in tables 5 and 6 in LBL experiments T2K and $\mathrm{NO} \nu \mathrm{A}$ at $95 \% \mathrm{CL}$ of statistical significance. The lower bounds are presented on the UV scale $\Lambda$ in each operator, when the corresponding Wilson coefficient is set to unity. Correspondingly, the upper constraints on the respective Wilson coefficients are shown when the scale is set to $\Lambda=1 \mathrm{TeV}$.

\subsection{Constraints on a single operator}

Treating the Wilson coefficients and the UV scale $\Lambda$ as arbitrary parameters, the nonobservation of new physics in the neutrino oscillation experiments can be used to derive constraints. In this section, we simulate the oscillation data that has recently been gathered in the LBL experiments $\mathrm{T} 2 \mathrm{~K}$ and $\mathrm{NO} \nu \mathrm{A}$ and the reactor experiments Daya Bay, Double Chooz and RENO to extract the experimental sensitivities to each operator.

We first present the constraints on the Wilson coefficient and the new physics scale $\Lambda$ for each operator individually in figure 8 . The constraints are provided at 95\% CL of statistical significance for 16 different operators using the neutrino oscillation data from the LBL experiments $\mathrm{T} 2 \mathrm{~K}$ and $\mathrm{NO} \nu \mathrm{A}$. The oscillations in these experiments are influenced through the pion decay process as discussed in section 2. The upper panel shows the lower bound on the $\Lambda$ in the LBL experiments when the Wilson coefficient of the corresponding operator is set to unity. Correspondingly, the upper bounds on the Wilson coefficients shown in the lower panel were obtained by keeping the scale parameter at $\Lambda=1 \mathrm{TeV}$. The results are presented for both experiments individually as well as for the combined bound of $\mathrm{T} 2 \mathrm{~K}$ and $\mathrm{NO} \nu \mathrm{A}$. Owing mainly to the higher fiducial mass in the near detector, we remark that $\mathrm{NO} \nu \mathrm{A}$ places the most restrictive constraint of the two experiments.

Note that in figure 8, the type-A dimension-6 SMEFT operators discussed in section 3 are in general most stringently constrained from the neutrino NSI parameters. This is what one would expect since these operators contribute directly to the Wilson coefficients $\epsilon_{L, R, S, P, T}$ in the LEFT unless one gets a suppression factor from the off-diagonal elements 


\begin{tabular}{|c|c|c|c|}
\hline Operator & $\mathrm{T} 2 \mathrm{~K}$ & $\mathrm{NO} \nu \mathrm{A}$ & Combined \\
\hline $\begin{array}{r}\mathcal{O}_{l e d q} \\
1211\end{array}$ & 6.4 & 19.8 & 19.8 \\
\hline $\begin{array}{c}\mathcal{O}_{l e q u}^{(1)} \\
1211\end{array}$ & 6.7 & 8.0 & 8.8 \\
\hline $\begin{array}{r}\mathcal{O}_{l e q u}^{(1)} \\
{ }_{2211}\end{array}$ & 6.6 & 8.0 & 8.8 \\
\hline $\begin{array}{r}\mathcal{O}_{l e d q} \\
2211\end{array}$ & 2.9 & 9.4 & 9.4 \\
\hline $\begin{array}{r}\mathcal{O}_{\text {lequ }}^{(3)} \\
1211\end{array}$ & 6.5 & 7.8 & 8.6 \\
\hline $\begin{array}{r}\mathcal{O}_{l e d q} \\
1212\end{array}$ & 3.1 & 3.7 & 4.1 \\
\hline $\begin{array}{r}\mathcal{O}_{l e d q} \\
2212\end{array}$ & 2.9 & 9.4 & 9.4 \\
\hline $\begin{array}{c}\mathcal{O}_{\text {lequ }}^{(3)} \\
\quad 2211\end{array}$ & 3.1 & 3.7 & 4.1 \\
\hline $\begin{array}{c}\mathcal{O}_{l q}^{(3)} \\
\quad 1211\end{array}$ & 1.2 & 10.4 & 10.4 \\
\hline $\begin{array}{c}\mathcal{O}_{l q}^{(3)} \\
\quad 2211\end{array}$ & 0.7 & 1.8 & 1.8 \\
\hline $\begin{array}{c}\mathcal{O}_{H q}^{(3)} \\
11\end{array}$ & 1.3 & 1.5 & 1.7 \\
\hline $\mathcal{O}_{H l}^{(3)}$ & 0.6 & 0.7 & 0.8 \\
\hline $\mathcal{O}_{H u d}$ & 1.2 & 1.5 & 1.7 \\
\hline $\mathcal{O}_{12 l}^{l l}$ & 1.2 & 1.4 & 1.6 \\
\hline $\begin{array}{c}\mathcal{O}^{(3)} \\
\quad 1212\end{array}$ & 0.9 & 1.0 & 1.1 \\
\hline $\begin{array}{c}\mathcal{O}_{l q}^{(3)} \\
\quad 2212\end{array}$ & 0.9 & 1.0 & 1.1 \\
\hline
\end{tabular}

Table 5. Lower bounds on the UV scale $\Lambda$ as obtained from the long-baseline neutrino experiment data. The results are arranged from strongest to weakest. The constraints are provided in units of $\mathrm{TeV}$ at $95 \% \mathrm{CL}$ significance for $\mathrm{T} 2 \mathrm{~K}$ and $\mathrm{NO} \nu \mathrm{A}$, both individually and combined.

of the CKM matrix as is the case for the $\mathcal{O}_{\text {ledq }}$ operator. In contrast, the type-B operators are relatively less constrained since they contribute to neutrino NSI parameters indirectly through modifying the interacting strength between gauge bosons and fermions at the weak scale $\mu=m_{W}$. Furthermore, among all the type-A operators, $\mathcal{O}_{\text {lequ }}^{(1)}$ and $\mathcal{O}_{\text {ledq }}^{(1)}$ dominate over all other operators for the following two reasons: (1) There is no off-diagonal CKM matrix element suppression in the quark sector; (2) For LBL experiments through pion decay, the dominant contributions to the neutrino NSI parameters come from the pseudoscalar interacting term $\epsilon_{P}$ in the LEFT defined in eq. (2.3), which can be clearly understood 


\begin{tabular}{|c|c|c|c|}
\hline Operator & $\mathrm{T} 2 \mathrm{~K}$ & $\mathrm{NO} \nu \mathrm{A}$ & Combined \\
\hline $\begin{array}{r}\mathcal{C}_{\text {ledq }} \\
1211\end{array}$ & $2.6 \times 10^{-8}$ & $2.8 \times 10^{-9}$ & $2.8 \times 10^{-9}$ \\
\hline $\begin{array}{c}\mathcal{C}_{\text {lequ }}^{(1)} \\
1211\end{array}$ & $2.4 \times 10^{-8}$ & $8.0 \times 10^{-8}$ & $8.8 \times 10^{-8}$ \\
\hline $\begin{array}{c}\mathcal{C}_{\text {lequ }}^{(1)} \\
2211\end{array}$ & $2.4 \times 10^{-8}$ & $2.8 \times 10^{-9}$ & $2.8 \times 10^{-9}$ \\
\hline $\begin{array}{r}\mathcal{C}_{\text {ledq }} \\
2211\end{array}$ & $1.3 \times 10^{-7}$ & $1.2 \times 10^{-8}$ & $1.2 \times 10^{-8}$ \\
\hline $\begin{array}{c}\mathcal{C}_{\text {lequ }}^{(3)} \\
1211\end{array}$ & $2.5 \times 10^{-8}$ & $1.7 \times 10^{-8}$ & $1.4 \times 10^{-8}$ \\
\hline $\begin{array}{r}\mathcal{C}_{\text {ledq }} \\
1212\end{array}$ & $1.1 \times 10^{-7}$ & $7.6 \times 10^{-8}$ & $6.2 \times 10^{-8}$ \\
\hline $\begin{array}{r}\mathcal{C}_{\text {ledq }} \\
2212\end{array}$ & $1.6 \times 10^{-7}$ & $1.7 \times 10^{-8}$ & $1.7 \times 10^{-8}$ \\
\hline $\begin{array}{c}\mathcal{C}_{\text {lequ }}^{(3)} \\
2211\end{array}$ & $1.5 \times 10^{-7}$ & $1.0 \times 10^{-7}$ & $8.5 \times 10^{-8}$ \\
\hline $\begin{array}{c}\mathcal{C}_{l q}^{(3)} \\
1211\end{array}$ & $6.3 \times 10^{-7}$ & $6.8 \times 10^{-8}$ & $6.6 \times 10^{-8}$ \\
\hline $\begin{array}{c}\mathcal{C}_{l q}^{(3)} \\
2211\end{array}$ & $2.8 \times 10^{-6}$ & $2.8 \times 10^{-7}$ & $2.8 \times 10^{-7}$ \\
\hline $\mathcal{C}_{11}^{(3)}$ & $5.8 \times 10^{-7}$ & $4.0 \times 10^{-7}$ & $3.3 \times 10^{-7}$ \\
\hline $\mathcal{C}_{11}^{(3)}$ & $2.5 \times 10^{-6}$ & $1.7 \times 10^{-6}$ & $1.4 \times 10^{-6}$ \\
\hline $\mathcal{C}_{H_{11}}$ & $6.0 \times 10^{-7}$ & $4.2 \times 10^{-7}$ & $3.5 \times 10^{-7}$ \\
\hline $\mathcal{C}_{1221}^{l l}$ & $6.6 \times 10^{-7}$ & $4.6 \times 10^{-7}$ & $3.8 \times 10^{-7}$ \\
\hline $\begin{array}{c}\mathcal{C}_{l q}^{(3)} \\
1212\end{array}$ & $1.3 \times 10^{-6}$ & $8.7 \times 10^{-7}$ & $7.2 \times 10^{-7}$ \\
\hline $\begin{array}{c}\mathcal{C}_{l q}^{(3)} \\
2212\end{array}$ & $1.3 \times 10^{-6}$ & $8.7 \times 10^{-7}$ & $7.2 \times 10^{-7}$ \\
\hline
\end{tabular}

Table 6. Upper bounds on the Wilson coefficient $\mathcal{C}$ as obtained from the long-baseline neutrino experiment data. The constraints are provided in units of $\mathrm{TeV}$ at $95 \% \mathrm{CL}$ significance for $\mathrm{T} 2 \mathrm{~K}$ and $\mathrm{NO} \nu \mathrm{A}$, both individually and combined.

from eq. (2.6) due to the enhancement factor $m_{\pi}^{2} /\left[m_{\mu}\left(m_{u}+m_{d}\right)\right]$.

Similarly, the constraints from the reactor antineutrino experiments Daya Bay, Double Chooz and RENO are shown in figure 9, also presented at 95\% CL for each of the considered reactor experiments. A combined bound from all the three experiments is also provided for each operator. We implement the non-standard physics through the beta decay and inverse beta decay processes, which are both present in all the three reactor experiments. Of the three reactor experiments, Day Bay and RENO impose similar limits to the observables, while Double Chooz presents somewhat weaker contribution due to its smaller exposure. 

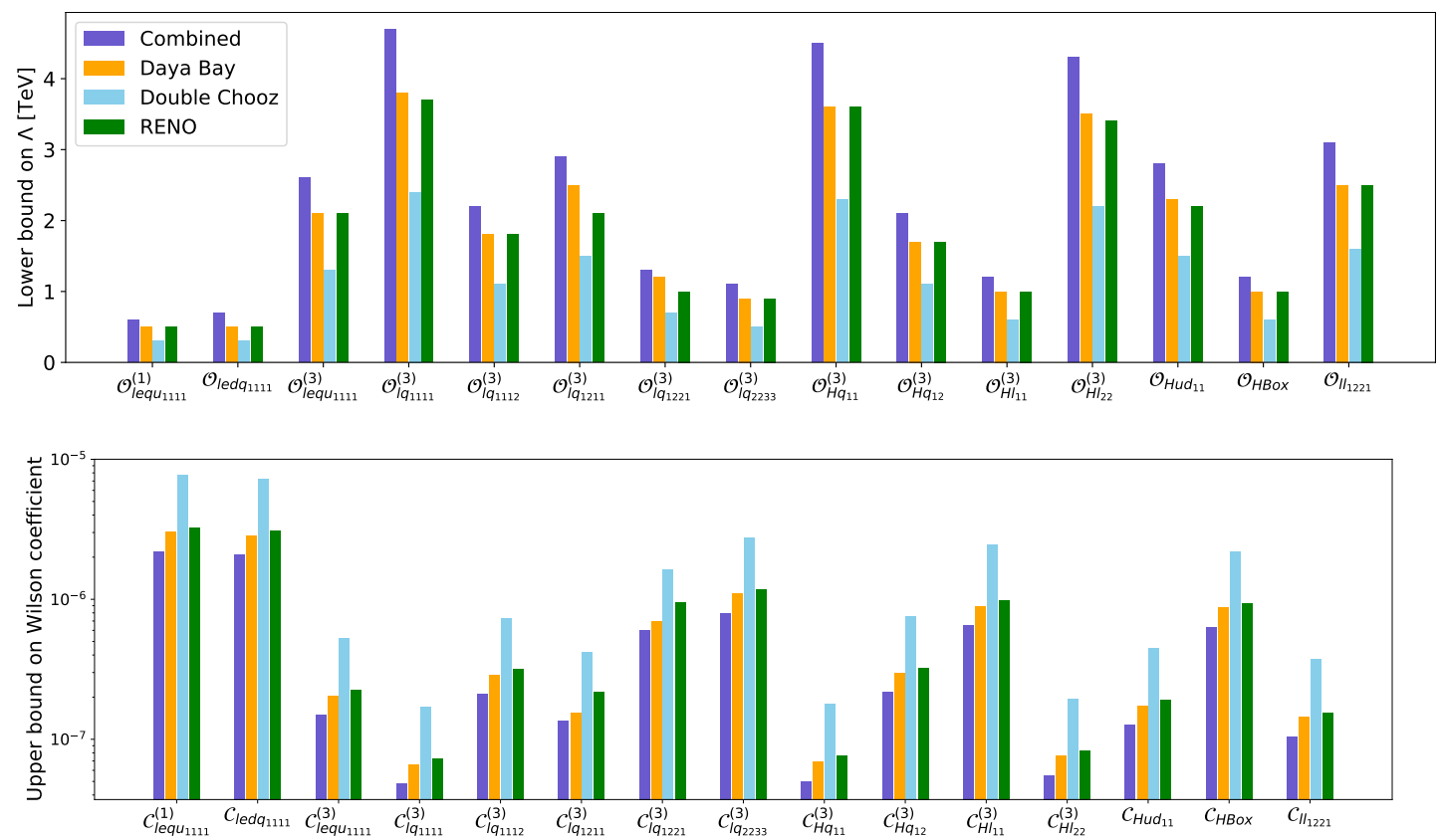

Figure 9. Constraints on part of dimension- 6 operators in tables 7 and 8 in reactor neutrino experiments Daya Bay, Double Chooz and RENO. The new physics scale $\Lambda$ associated with a specific operator is constrained from below and the corresponding Wilson coefficient from above when $\Lambda=1 \mathrm{TeV}$. The results are obtained at 95\% CL.

Different from the LBL neutrino experiments where the neutrino NSI parameters are sensitive to the pseudo-scalar interaction, the reactor neutrino experiments are dominated by the vector and axial-vector type interactions described by the $\epsilon_{L, R}$ terms in eq. (2.3). This can also be understood by looking at the matching formulas in eqs. (2.4)-(2.5) and explains why $\mathcal{O}_{l q}^{(3)}, \mathcal{O}_{H q}^{(3)}$, and $\mathcal{O}_{22}^{(3)}$ are more constrained than all the other operators listed in figure 9. Furthermore, (1) the type-A operator $\mathcal{O}_{11}^{(3)}$ dominates over the type-B operators $\mathcal{O}_{\mathrm{Hq}}^{(3)}$ and $\mathcal{O}_{22}^{(3)}$ for the same reason as explained for the LBL neutrino experiments; (2) $\mathcal{O}_{2 l}^{(3)}$ is more constrained compared with $\mathcal{O}_{H l}^{(3)}$ since the second generation charged lepton is heavier than the first one, thus its contribution to the corresponding NSI parameter is less suppressed than that from $\mathcal{O}_{{ }_{11}}^{(3)}$.

Comparing figures 8 and 9 , one notices that the results extracted from the LBL neutrino oscillation experiments are generally more restrictive compared to those obtained from the reactor experiments. While the strongest bound on the UV scale $\Lambda$ is nearly $20 \mathrm{TeV}$ from the LBL experiments, the most restrictive bound from the reactor experiments is about $4 \mathrm{TeV}$. However, it should be emphasized that the results following from the LBL and the reactor experiments are mostly sensitive to different operators. This highlights the complementary nature that lies between the LBL and the reactor experiments.

We also point out that, for certain operators, the upper bounds provided for the Wilson coefficients in figures 8 and 9 are sensitive to the UV scale $\Lambda$. Among all the dimension- 6 


\begin{tabular}{|c|c|c|c|c|}
\hline Operator & Daya Bay & Double Chooz & RENO & Combined \\
\hline $\begin{array}{c}\mathcal{O}_{l e q u}^{(1)} \\
\quad 1111\end{array}$ & 0.5 & 0.3 & 0.5 & 0.6 \\
\hline $\begin{array}{r}\mathcal{O}_{l e d q} \\
\quad 1111\end{array}$ & 0.5 & 0.3 & 0.5 & 0.7 \\
\hline $\mathcal{O}_{\text {lequ }}^{(3)}$ & 2.1 & 0.3 & 2.1 & 2.6 \\
\hline $\begin{array}{c}\mathcal{O}^{(3)} \\
\quad 1111\end{array}$ & 3.8 & 2.4 & 3.7 & 4.7 \\
\hline $\begin{array}{c}\mathcal{O}_{l q}^{(3)} \\
\quad 1112\end{array}$ & 1.8 & 1.1 & 1.8 & 2.2 \\
\hline $\begin{array}{c}\mathcal{O}_{l q}^{(3)} \\
\quad l 211\end{array}$ & 2.5 & 1.5 & 2.1 & 2.9 \\
\hline $\begin{array}{c}\mathcal{O}_{l q}^{(3)} \\
\quad l 221\end{array}$ & 1.2 & 0.7 & 1.0 & 1.3 \\
\hline $\begin{array}{c}\mathcal{O}_{l q}^{(3)} \\
\quad 2233\end{array}$ & 0.9 & 0.5 & 0.9 & 1.1 \\
\hline $\mathcal{O}_{H q}^{(3)}$ & 3.6 & 2.3 & 3.6 & 4.5 \\
\hline $\begin{array}{c}\mathcal{O}_{H q}^{(3)} \\
{ }_{12}\end{array}$ & 1.7 & 1.1 & 1.7 & 2.1 \\
\hline $\mathcal{O}_{H 1}^{(3)}$ & 1.0 & 0.6 & 1.0 & 1.2 \\
\hline $\mathcal{O}_{{ }_{22}}^{(3)}$ & 3.5 & 2.2 & 3.4 & 4.3 \\
\hline $\mathcal{O}_{H u d}$ & 2.3 & 1.5 & 2.2 & 2.8 \\
\hline $\mathcal{O}_{H \square}$ & 1.0 & 0.6 & 1.0 & 1.2 \\
\hline $\mathcal{O}_{1221}^{l l}$ & 2.5 & 1.6 & 2.5 & 3.1 \\
\hline
\end{tabular}

Table 7. Lower bounds on the UV scale $\Lambda$ as obtained from the reactor neutrino experiment data. The constraints are provided in units of $\mathrm{TeV}$ at $95 \%$ CL significance for Daya Bay, Double Chooz and RENO, both individually and combined.

SMEFT operators that contribute dominantly to neutrino NSI parameters, we find that there are only three operators whose Wilson coefficients are sensitive to $\Lambda$. We show them in the left and the right panels of figure 10 for the LBL and the reactor neutrino experiments respectively for $\Lambda$ in the $[1,10] \mathrm{TeV}$ range. ${ }^{11}$ In addition, for these three operators, we also observe that their phases oscillate very rapidly as a function of $\Lambda$, implying $C P$-violating NSI phases that we further explore in section 7.

\footnotetext{
${ }^{11}$ As one may see from the results shown in figure 10 it still holds that the low-energy effects are approximately independent from the UV scale $\Lambda$.
} 


\begin{tabular}{|c|c|c|c|c|}
\hline Operator & Daya Bay & Double Chooz & RENO & Combined \\
\hline $\mathcal{C}_{\text {lequ }}^{(1)}{ }_{1111}$ & $3.0 \times 10^{-6}$ & $7.8 \times 10^{-6}$ & $3.2 \times 10^{-6}$ & $2.2 \times 10^{-6}$ \\
\hline $\begin{array}{r}\mathcal{C}_{\text {ledq }} \\
\quad 1111\end{array}$ & $2.8 \times 10^{-6}$ & $7.2 \times 10^{-6}$ & $3.1 \times 10^{-6}$ & $2.1 \times 10^{-6}$ \\
\hline $\begin{array}{c}\mathcal{C}_{\text {lequ }}^{(3)} \\
1111\end{array}$ & $2.0 \times 10^{-7}$ & $5.2 \times 10^{-7}$ & $2.2 \times 10^{-7}$ & $1.5 \times 10^{-7}$ \\
\hline $\begin{array}{c}\mathcal{C}_{l q}^{(3)} \\
1111\end{array}$ & $6.6 \times 10^{-8}$ & $1.7 \times 10^{-7}$ & $7.2 \times 10^{-8}$ & $4.8 \times 10^{-8}$ \\
\hline $\begin{array}{c}\mathcal{C}_{l q}^{(3)} \\
1112\end{array}$ & $2.9 \times 10^{-7}$ & $7.2 \times 10^{-7}$ & $3.2 \times 10^{-7}$ & $2.1 \times 10^{-7}$ \\
\hline $\begin{array}{c}\mathcal{C}_{l q}^{(3)} \\
1211\end{array}$ & $1.5 \times 10^{-7}$ & $4.2 \times 10^{-7}$ & $2.2 \times 10^{-7}$ & $1.3 \times 10^{-7}$ \\
\hline $\begin{array}{c}\mathcal{C}_{l q}^{(3)} \\
1221\end{array}$ & $6.9 \times 10^{-7}$ & $1.6 \times 10^{-6}$ & $9.5 \times 10^{-7}$ & $6.0 \times 10^{-7}$ \\
\hline $\begin{array}{c}\mathcal{C}_{l q}^{(3)} \\
2233\end{array}$ & $1.1 \times 10^{-6}$ & $2.8 \times 10^{-6}$ & $1.2 \times 10^{-6}$ & $7.9 \times 10^{-7}$ \\
\hline $\mathcal{C}_{11}^{(3)}$ & $6.9 \times 10^{-8}$ & $1.8 \times 10^{-7}$ & $7.6 \times 10^{-8}$ & $5.0 \times 10^{-8}$ \\
\hline $\begin{array}{c}\mathcal{C}_{H q}^{(3)} \\
12\end{array}$ & $3.0 \times 10^{-7}$ & $7.6 \times 10^{-7}$ & $3.2 \times 10^{-7}$ & $2.2 \times 10^{-7}$ \\
\hline $\begin{array}{c}\mathcal{C}_{H l}^{(3)} \\
11\end{array}$ & $8.9 \times 10^{-7}$ & $2.5 \times 10^{-6}$ & $9.8 \times 10^{-7}$ & $6.5 \times 10^{-7}$ \\
\hline $\mathcal{C}_{22}^{(3)}$ & $7.6 \times 10^{-8}$ & $1.9 \times 10^{-7}$ & $8.3 \times 10^{-8}$ & $5.5 \times 10^{-8}$ \\
\hline $\mathcal{C}_{H u d}$ & $1.7 \times 10^{-7}$ & $4.5 \times 10^{-7}$ & $1.9 \times 10^{-7}$ & $1.3 \times 10^{-7}$ \\
\hline $\mathcal{C}_{H \square}$ & $8.7 \times 10^{-7}$ & $2.2 \times 10^{-6}$ & $9.3 \times 10^{-7}$ & $6.3 \times 10^{-7}$ \\
\hline $\mathcal{C}_{1221}^{l l}$ & $1.4 \times 10^{-7}$ & $3.7 \times 10^{-7}$ & $1.5 \times 10^{-7}$ & $1.0 \times 10^{-7}$ \\
\hline
\end{tabular}

Table 8. Upper bounds on the Wilson coefficient $\mathcal{C}$ as obtained from the reactor neutrino experiment data. The constraints are provided in units of $\mathrm{TeV}$ at $95 \%$ CL significance for Daya Bay, Double Chooz and RENO, both individually and combined.

\subsection{Constraints on multiple operators}

Constraints on the Wilson coefficients could change when more than one dimension-6 SMEFT operator is present at the UV scale, as is the case for the simplified scalar leptoquark model discussed in section 5 . The change is due to the operator mixing through the RGEs. As a result, the existence of multiple SMEFT operators may contribute simultaneously to the neutrino NSI parameters at the low energy scale, such that the sensitivities to individual Wilson coefficients also change as the other Wilson coefficients may produce a similar imprint on the neutrino oscillation data. The correlation among different dimension-6 SMEFT operator at the UV scale $\Lambda$ is absent in the case presented in last subsection, where we only consider one non-vanishing dimension-6 SMEFT operator at a 

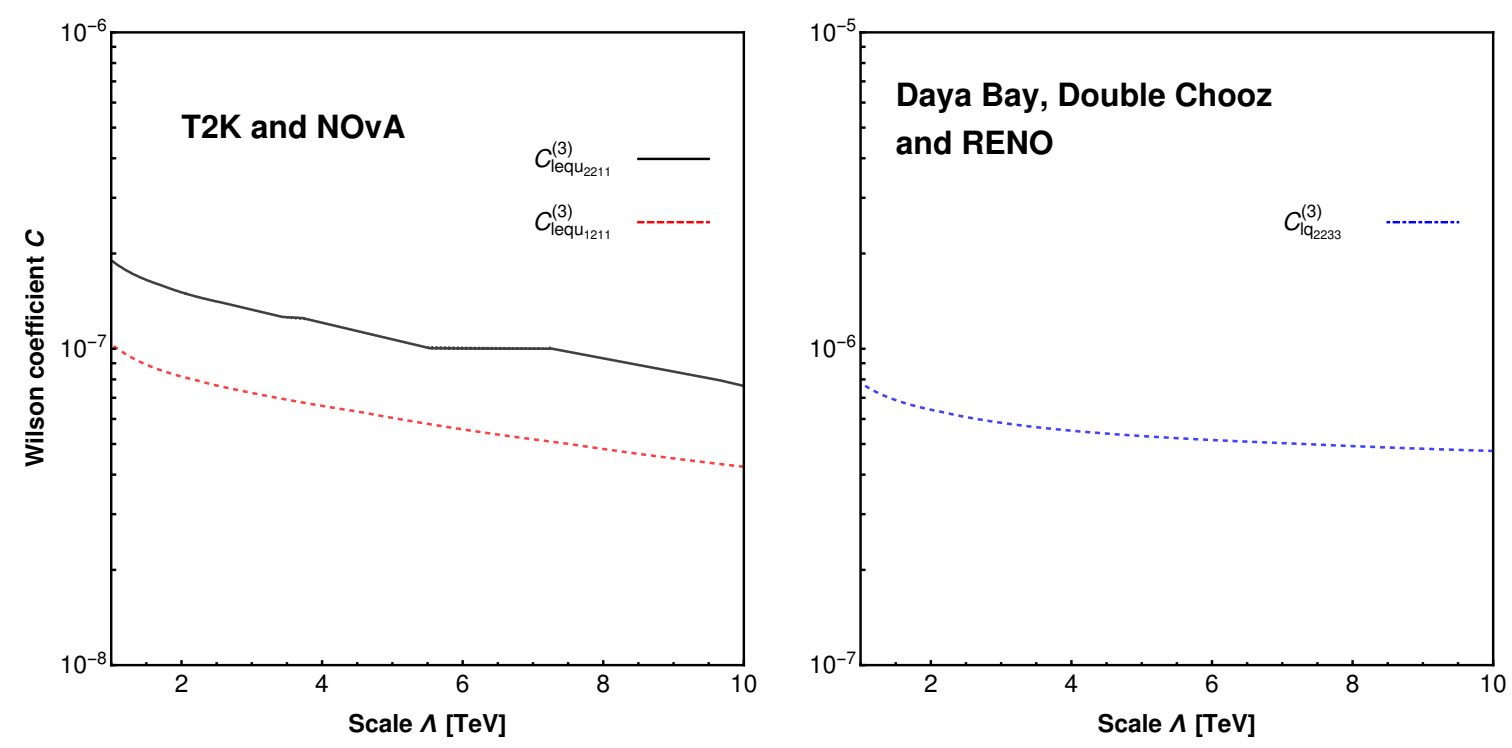

Figure 10. Upper constraints on Wilson coefficient as function of the scale $\Lambda$. The left panel shows the scale-dependence for operators in $\mathrm{LBL}$ experiments $\mathrm{T} 2 \mathrm{~K}$ and $\mathrm{NO} \nu \mathrm{A}$ whilst the right panel represents the reactor experiments Daya Bay, Double Chooz and RENO. The upper constraints on the Wilson coefficients are obtained at 95\% CL.

time at the UV scale. Our discussion on the correlation among different operators at the UV scale shall not be confused with the study of a subset of SMEFT operators discussed in refs. [66, 82], where one only focuses on a closed subset of the full RGEs related to the observables under the study in the latter case. As we shall see later in this subsection, the correlation we study here is in general important and shall be considered for a relatively more rigorous study.

Without losing generality, we assume that all the dimension-6 SMEFT operators are associated with the same high-energy scale $\Lambda$ and then present the interference of multiple operators in figure 11. The effects, resulting from the correlation among different operators, on the upper bounds of two Wilson coefficients are shown in the left (right) panel for LBL (reactor) experiments. The shaded areas indicate the allowed values of two Wilson coefficients at $95 \% \mathrm{CL}$ statistical significance when no other operators are present. The scale is set at $\Lambda=1 \mathrm{TeV}$. The operators selected for this example represent both the typeA and the type-B classes, but they generate similar source and detection NSI parameter values. As one can see from the resulting sensitivities, adding a second Wilson coefficient decreases the sensitivities to both Wilson coefficients as opposed to if they were assumed to be the single non-vanishing ones.

To see the quantitative impact of extra operators on the upper bound of the Wilson coefficient, we can, for example, compare the difference between the upper bound for $C_{\text {ledq }}$ when only one dimension-6 SMEFT operator is considered at the input scale and that when multiple operators exist at the UV scale. In the former case, as one can see from the lower panel of figure 8, its upper bound from the combined analysis is $C_{2211} \lesssim 10^{-8}$; while 

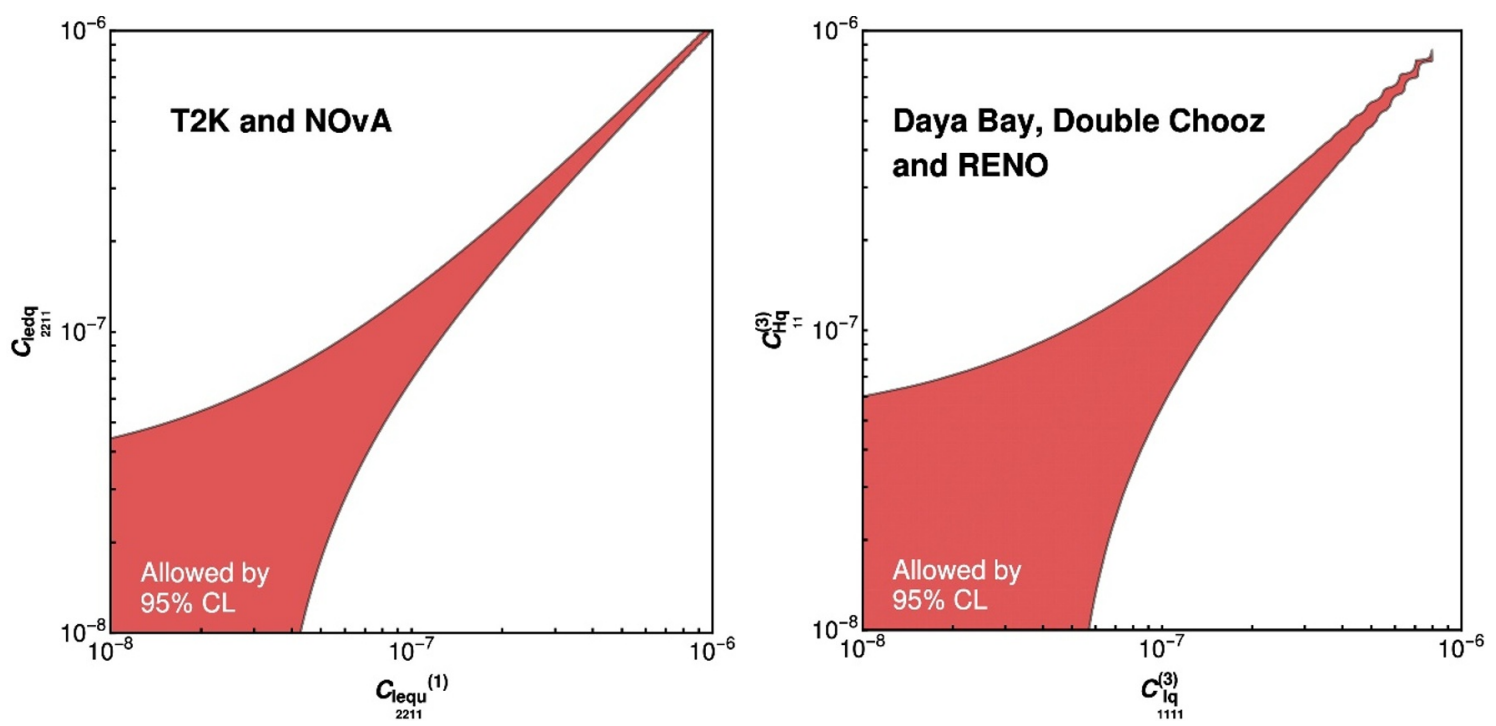

Figure 11. Left panel: upper constraints on the Wilson coefficients corresponding to the operators $\left(\bar{\ell}_{2} e_{2}\right) \varepsilon_{j k}\left(\bar{q}_{1} u_{1}\right)$ and $\left(\bar{\ell}_{2} e_{2}\right)\left(\bar{d}_{1} q_{1}\right)$ with $\Lambda=1 \mathrm{TeV}$. The constraints are obtained for the LBL experiments T2K and $\mathrm{NO} \nu \mathrm{A}$ at $95 \% \mathrm{CL}$ significance. Right panel: same as the left panel but with the application of Daya Bay, Double Chooz and RENO on operators $\left(\bar{\ell}_{1} \sigma_{\mu \nu} e_{1}\right) \varepsilon_{j k}\left(\bar{q}_{1} \sigma^{\mu \nu} u_{1}\right)$ and $\left(H^{\dagger} i \vec{D}_{\mu} H\right)\left(\bar{q}_{1} \tau^{I} \gamma^{\mu} q_{1}\right)$ instead.

in the latter case, according to the left panel of figure 11, it relaxes to $\lesssim 5 \times 10^{-8}$ when the Wilson coefficient $C_{\text {lequ }}^{(1)}$ also has a non-zero input at the UV scale $\Lambda$. Moreover if $C_{2211}$ happens to be roughly equal to $C_{2211}^{(1)}$, then both of them can be as large as $\sim 10^{-6}$ or even larger as indicated by the diagonal pin in the left panel of figure 11, the reason for this is that the two operators contributes opposite sign for the $\epsilon_{P}$ in the LEFT of which the effect is dominant in the LBL experiments. Similar behavior repeats in the reactor experiments, as demonstrated for coefficients $C_{l q}^{(3)}$ and $C_{11}^{(3)}$ in the right panel of figure 11.

We point out that, due to the correlation of multiple operators at the UV scale $\Lambda$, relaxation on the upper (lower) bounds of the Wilson coefficients (UV scale $\Lambda$ ) turns out to be a common feature from our study. However, we are not claiming that inclusion of extra operators will always lead to stronger (weaker) constraints on the Wilson coefficients (UV scale). One counterexample is shown in figure 12, where the left and the right panels are for LBL and reactor neutrino experiments respectively. Comparing with the upper bounds obtained when only one operator is considered at the UV scale $\Lambda$, inclusion of an extra operator leads to stronger constraints on all the operators shown in figure 12 .

Based on observation above, we claim that, even though it may be computationally challenging to include the correlation among all observable-related SMEFT operators at the UV scale in practice, one shall at least take into account the full correlation among different operators for specific UV models at the UV scale, as has been done for the simplified scalar leptoquark model in this work. 

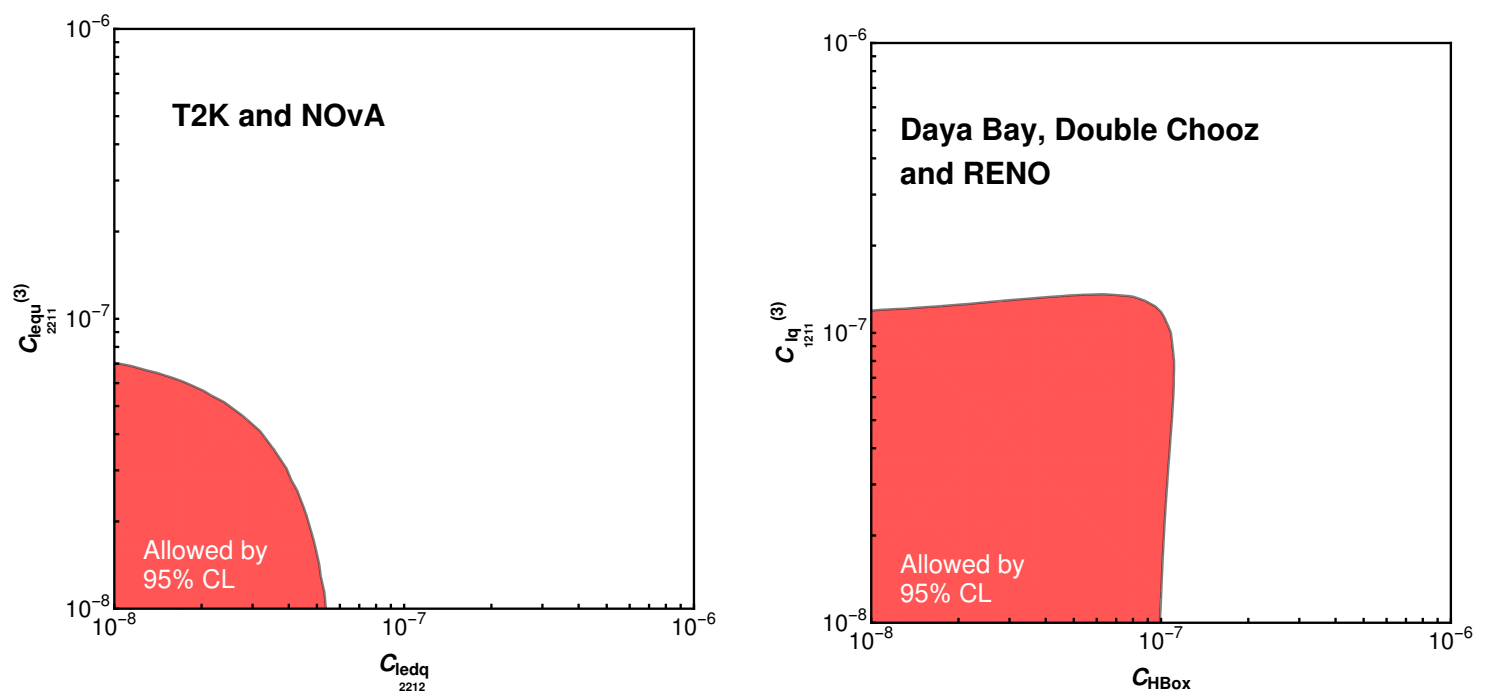

Figure 12. Left panel: upper constraints on the Wilson coefficients corresponding to the operators $\left(\bar{\ell}_{2} e_{2}\right) \varepsilon_{j k}\left(\bar{q}_{1} u_{1}\right)$ and $\left(\bar{\ell}_{2} e_{2}\right)\left(\bar{d}_{1} q_{1}\right)$ with $\Lambda=1 \mathrm{TeV}$. The constraints are obtained for the LBL experiments T2K and NO $\nu$ A at $95 \%$ CL significance. Right panel: same as the left panel but with the application of the reactor experiments Daya Bay, Double Chooz and RENO with operators $\left(\phi^{\dagger} \phi\right) \square\left(\phi^{\dagger} \phi\right)$ and $\left(\bar{\ell}_{1}^{j} \sigma_{\mu \nu} e_{1}\right) \varepsilon_{j k}\left(\bar{q}_{1}^{k} \sigma^{\mu \nu} u_{1}\right)$ instead.

\section{$7 \quad C P$ violation from NSI phases}

One of the primary objectives in the on-going and future neutrino oscillation experiments is to investigate whether there exists $C P$ violation in the leptonic sector. One possible source of $C P$ violation is the Dirac $\mathrm{CP}$ phase $\delta_{C P}$, which corresponds to either $C P$-conserving when $\sin \delta_{C P}=0$ or $C P$-violating when $\sin \delta_{C P} \neq 0$. Such $C P$ violation may also emerge due to the neutrino NSIs in the source or the detector. The importance of studying $C P$ violation in the NSI parameters has been highlighted in ref. [16]. It has been shown that $C P$-violating NSI phases may resolve tensions in experiments $[83,84]$ as well as disrupt future experimental programs [85]. In this section, we set focus on the new NSI phases that could be generated by dimension- 6 operators in SMEFT.

Using the Wilson package and the matching method discussed in sections 2 and 3, we are able to find two operators that could generate a $C P$-violating NSI phase in LBL experiments such as $\mathrm{T} 2 \mathrm{~K}$ and $\mathrm{NO} \nu \mathrm{A}$, where neutrino production is based on pion decay, and one in reactor experiments like Daya Bay, Double Chooz and RENO, where both processes based on beta decays and inverse beta decays occur. We calculate the values that would be acquired in the source and detection NSI parameters $\epsilon_{\mu e}^{s}$ and $\epsilon_{\mu e}^{d}$ in the event where operators $\mathcal{O}_{l e d q}$ and $\mathcal{O}_{l q}^{(3)}$ are considered at the UV scale $\Lambda$ in the LBL neutrino experiments and operator $\mathcal{O}_{12}^{(3)}{ }_{122}^{1212}$ in the reactor neutrino experiments, respectively. ${ }^{12} \mathrm{We}$ also study the NSI parameter values obtained for the $C P$-conserving operators $\mathcal{O}_{\text {lequ }}^{(1)}$ and

\footnotetext{
${ }^{12}$ We also obtain similar values for $\epsilon_{e \mu}^{s}$ in beta decay.
} 

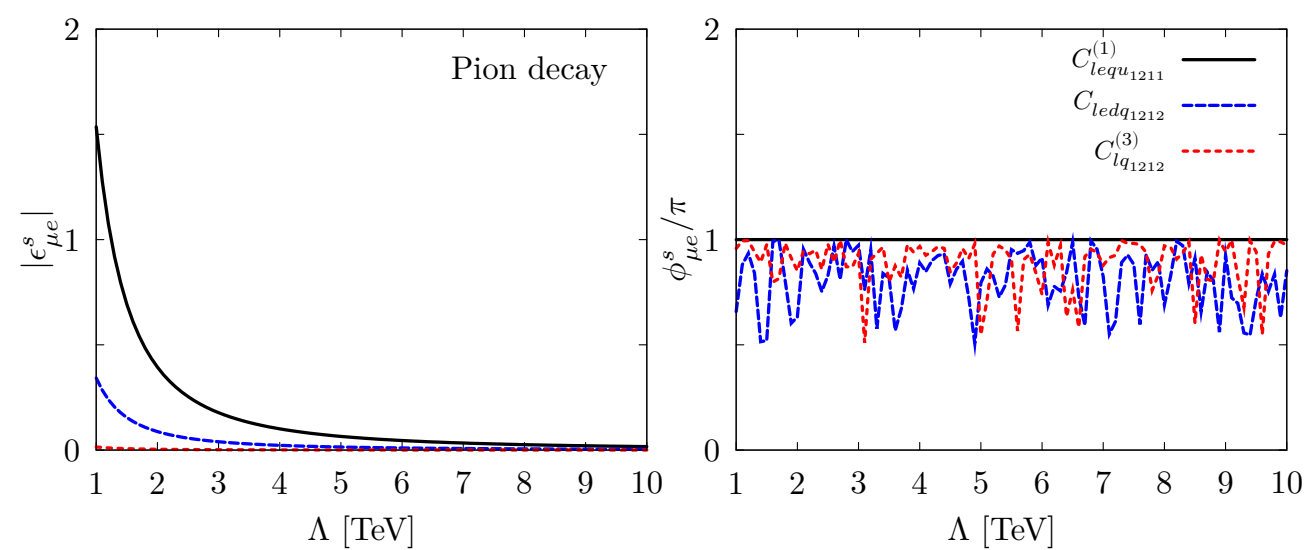

Figure 13. The magnitude and phase of the source NSI parameter $\epsilon_{\mu e}^{s}$ in pion-decay-driven neutrino oscillation experiments. The values obtained for the NSI parameter are shown for one $C P$-conserving operator (solid black) and two $C P$-violating operators (dashed blue and dotted red) as function of the scale $\Lambda$.
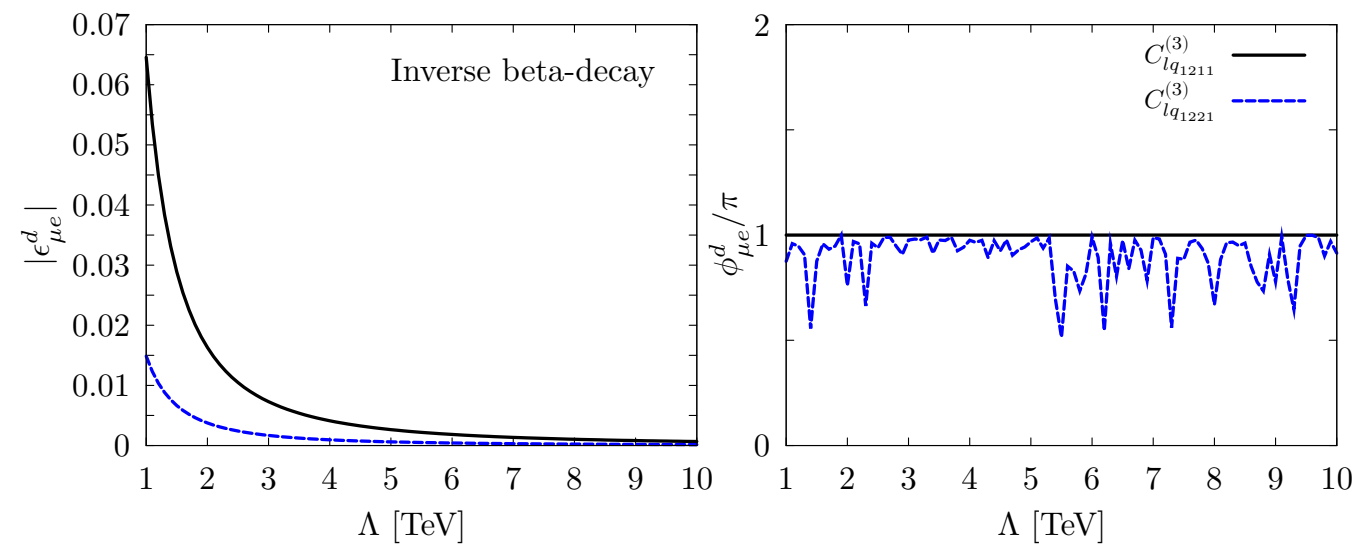

Figure 14. The magnitude and phase of the detection NSI parameter $\epsilon_{\mu e}^{d}$ in reactor experiments, where detection is based on inverse beta decay. As an example, the values acquired for the NSI parameter are presented for one $C P$-conserving operator (solid black) and one $C P$-violating operator (dashed blue) as function of the UV-scale $\Lambda$.

$\mathcal{O}_{l q}^{(3)}$. In each case we consider only one operator at a time. The source and detection NSI computed from individual operators are presented for LBL experiments in figure 13 and reactor experiments in figure 14, respectively.

It can be seen in the results presented in figure 13 that the operators $\mathcal{O}_{l e d q}$ and $\mathcal{O}_{12}^{(3)}$ lead to a sizeable value in the $C P$-violating NSI phase $\phi_{\mu e}^{s}$ in LBL experiments where neutrinos are produced via pion decay. On the other hand, it is evident in figure 14 that rather large $C P$-violating NSI phase $\phi_{\mu e}^{d}$ can be generated from operator $\mathcal{O}_{l q}^{(3)}$ in reactor neutrino experiments, where detection via inverse beta decay is prevalent. As an example of the $C P$-conserving operators, which we find all other operators in our consideration to 
fall into, are also present the NSI generated from operators $\mathcal{O}_{\text {lequ }}^{(1)}$ and $\mathcal{O}_{l q}^{(3)}$ in LBL and reactor experiments, respectively. The exact value of the NSI phase depends largely on the scale $\Lambda$, which we consider to be in the $[1,10] \mathrm{TeV}$ range.

One may ask whether the NSI generated from operators $\mathcal{O}_{l e d q}$ and $\mathcal{O}_{l q 12}^{(3)}$ can induce a noticeable signal in the neutrino oscillation experiments we consider in this work. Although the NSI phases pertaining to these operators can be $C P$-violating, the relatively small magnitude generated for these operators are below the scope of the present data available in $\mathrm{T} 2 \mathrm{~K}$ and $\mathrm{NO} \nu \mathrm{A}$. Respectively, the data obtained from the reactor neutrino experiments Daya Bay, Double Chooz and RENO suggests that the $C P$-violating NSI phase from operator $\mathcal{O}_{l q}^{(3)}$ is also below the present limit.

\section{Conclusions}

Ongoing neutrino oscillation experiments are providing more and more precise measurements on neutrino properties, which, on one hand, deepens our understanding of neutrinos, and on the other hand, opens the possibility to study neutrino NSIs more closely. SMEFT provides a systematic and model-independent method for this purpose. In this work, we study constraints on neutrino NSIs from neutrino experiments $\mathrm{T} 2 \mathrm{~K}, \mathrm{NO} \nu \mathrm{A}$, Daya Bay, Double Chooz and RENO in the SMEFT framework. The SMEFT operators are naturally defined at a UV scale $\Lambda$ that is larger than the scale where neutrino oscillation experiments operate. As a result, matching of different EFTs and the renormalization group running need to be taken into account at different scales. A schematic workflow of this procedure is depicted in figure 1, together with the dimension- 6 operators that contribute to neutrino NSIs under the corresponding EFT names.

A simplified scalar leptoquark model is adopted for the illustration of our workflow in the top-down EFT approach. At tree level, there are about 300 SMEFT operators that are related to neutrino NSIs at the UV scale. At a different scale, the SMEFT operators mix through the anomalous dimension matrix, and as a consequence, all SMEFT operators could contribute to the neutrino NSI parameters at the $2 \mathrm{GeV}$ scale. Using presently available upper bounds on the NSI parameters from neutrino oscillation experiments, we investigate how these experiments can be used to constrain this simplified model. We find that the current upper bound on $\left|\epsilon_{\mu e}^{s}\right|$ from pion decay at the LBL experiments T2K and $\mathrm{NO} \nu \mathrm{A}$ lead to the most stringent constraint on the simplified model as shown in figures 3 and 5. Furthermore, we conclude that constraints from the charged current neutrino NSI parameters are much stronger than those obtained from collider studies at the ATLAS and the CMS, implying complementarity between low and high-energy experiments in searching for new physics.

We also generalize our study to obtain the constraints on individual dimension-6 SMEFT operators from neutrino oscillation experiments in the bottom-up approach, where only one dimension-6 SMEFT operator is assumed to have a non-vanishing value at the UV scale. The results are shown in figure 8 and figure 9 for LBL and reactor neutrino experiments respectively. We find that LBL neutrino experiments $\mathrm{T} 2 \mathrm{~K}$ and $\mathrm{NO} \nu \mathrm{A}$ are 
already sensitive to new physics around $20 \mathrm{TeV}$ by fixing the Wilson coefficients at unity, as indicated by the $\mathcal{O}_{\text {lequ }}^{(1)}$ and the $\mathcal{O}_{\text {lequ }}^{(1)}$ operators in the upper panel of figure 8 . In contrast, reactor neutrino experiments Daya Bay, Double Chooz and RENO are now reaching the $5 \mathrm{TeV}$ range as implied by the $\mathcal{O}_{l q}^{(3)}, \mathcal{O}_{H q}^{(3)}$ and $\mathcal{O}_{\frac{H 2}{(1)}}^{(3)}$ operators in the upper panel of figure 9. Though LBL neutrino experiments impose generally much stronger constraints on the SMEFT operators than the reactor neutrino experiments, the two experiment groups are mainly sensitive to different sets of SMEFT operators, which highlights the complementarity between LBL and reactor neutrino experiments.

The correlation among the different dimension- 6 operators becomes important when more than one operator is studied at the same time. In section 6.2 , effects from the correlation among multiple operators are studied. The results are obtained by considering two non-vanishing dimension-6 SMEFT operators at the same time at the UV scale $\Lambda$. The effect from this correlation is presented in figures 11 and 12 for both LBL and reactor neutrino experiments. We find that the correlation among different operators at the UV scale could weaken the constraints on SMEFT operators by several orders of magnitude as shown in figure 11. We point out that it is also possible that stronger constraints on the SMEFT operators could be obtained with the inclusion of multiple operator correlation, as is shown in figure 12. Basing on this observation, we conclude our work with the following remark: though it may be technically challenging to include the correlation among all observable-related SMEFT operators at the UV scale, one shall at least take the correlation among all operators that are induced by a specific UV model into account as is studied for the simplified scalar leptoquark model in section 5.

We also examine whether new sources of $C P$ violation can be found at the UV scale and identify several operators for which considerable NSI phases could be generated, with three such operators showcased in figures 13 and 14. The magnitude of the relevant neutrino NSI is however found to fall below the sensitivities of the present data.

\section{Acknowledgments}

We thank Adam Falkowski and Gang Li for helpful discussion, the HPC Cluster of ITPCAS for the computing support and TseChun Wang for his contribution in the early stage of this project. YD, HLL and JHY were supported by the National Science Foundation of China (NSFC) under Grants No. 11875003 and No. 12022514. JT and SV were supported in part by National Natural Science Foundation of China under Grant Nos. 12075326, 11505301 and 11881240247, Guangdong Basic and Applied Basic Research Foundation under Grant No. 2019A1515012216. SV was also supported by China Postdoctoral Science Foundation under Grant No. 2020M672930. YD was also supported in part under U.S. Department of Energy contract DE-SC0011095. JHY was also supported by the National Science Foundation of China (NSFC) under Grants No. 11947302. JT also acknowledges the support from the CAS Center for Excellence in Particle Physics (CCEPP). 


\section{A LEFT for muon decay and the matching between QFT and QM for- malisms}

In this section, we present an example to show the details of the matching between the QFT and the QM formalisms of neutrino NSIs following the procedure in ref. [20]. Since only one neutrino is involved in pion decay, as well as in beta decay and inverse beta decay, the matching procedure is relatively straightforward and we reproduced the matching formulae presented in ref. [20]. This procedure is less trivial in muon decay as the muon neutrino and electron antineutrino are produced simultaneously. We thus use muon decay as our example here for illustration. ${ }^{13}$

To start, we write the most general low-energy EFT describing muon decay as follows, with an introduction of the right-handed partner of neutrinos $[86-88],{ }^{14}$

$$
\begin{aligned}
\mathcal{L}_{\mu}=\frac{G_{\mathrm{F}}}{\sqrt{2}}\{ & h_{11}(s+p)_{e \nu_{e}}(s+p)_{\nu_{\mu} \mu}+h_{12}(s+p)(s-p)+h_{21}(s-p)(s+p)+h_{22}(s-p)(s-p) \\
& +g_{11}\left(v^{\alpha}+a^{\alpha}\right)_{e \nu_{e}}\left(v_{\alpha}+a_{\alpha}\right)_{\nu_{\mu} \mu}+g_{12}\left(v^{\alpha}+a^{\alpha}\right)\left(v_{\alpha}-a_{\alpha}\right)+g_{21}\left(v^{\alpha}-a^{\alpha}\right)\left(v_{\alpha}+a_{\alpha}\right) \\
& +\left(1+g_{22}\right)\left(v^{\alpha}-a^{\alpha}\right)\left(v_{\alpha}-a_{\alpha}\right)+f_{11}\left(t^{\alpha \beta}+u^{\alpha \beta}\right)_{e \nu_{e}}\left(t_{\alpha \beta}+u_{\alpha \beta}\right)_{\nu_{\mu} \mu} \\
& \left.+f_{22}\left(t^{\alpha \beta}-u^{\alpha \beta}\right)\left(t_{\alpha \beta}-u_{\alpha \beta}\right)+\text { h.c. }\right\},
\end{aligned}
$$

where

$$
s_{i j} \equiv \bar{u}_{i} u_{j}, p_{i j} \equiv \bar{u}_{i} \gamma_{5} u_{j}, v_{i j}^{\mu} \equiv \bar{u}_{i} \gamma^{\mu} u_{j}, a_{i j}^{\mu} \equiv \bar{u}_{i} \gamma^{\mu} \gamma_{5} u_{j}, t_{i j}^{\alpha \beta} \equiv \bar{u}_{i} \sigma^{\mu \nu} u_{j}, u_{i j}^{\alpha \beta} \equiv \bar{u}_{i} \sigma^{\mu \nu} \gamma_{5} u_{i} .
$$

For comparison, the relation between our notations and those in ref. [20], denoted as "FGT" in the following, takes the form

$$
g_{22}=\rho_{L}^{\mathrm{FGT}}, \quad h_{21}=-2 \rho_{R}^{\mathrm{FGT}}
$$

We denote the muon decay process as $\mu^{-}\left(p_{2}\right) \rightarrow \nu_{\mu}\left(p_{1}\right)+e^{-}\left(p_{3}\right)+\bar{\nu}_{e}\left(p_{4}\right)$, with their four-momenta specified explicitly. To obtain the matching between the Wilson coefficients in the LEFT above and the NSI parameters $\epsilon^{s, d}$, we follow the notations in ref. [20]. At linear level in the Wilson coefficients in $\mathcal{L}_{\mu}$, the master formula relevant for our discussion here can be written as [20]

$$
\epsilon_{\alpha \beta}^{s}=\sum_{X} p_{X L}\left[\epsilon_{X}\right]_{\alpha \beta}^{*},
$$

with the production coefficients $p_{X L}$ defined as

$$
p_{X L} \equiv \frac{\int d \Pi_{P^{\prime}} A_{X}^{P} \bar{A}_{L}^{P}}{\int d \Pi_{P^{\prime}} A_{L}^{P} \bar{A}_{L}^{P}}, \quad d \Pi_{P^{\prime}} \equiv \frac{d \Pi_{P}}{d E_{\nu}},
$$

\footnotetext{
${ }^{13}$ We thank Martín González-Alonso for bringing their published version of ref. [20] to our attention, where they also discussed the matching for muon decay. We also thank Martín for pointing out the disagreement between our previous results presented in this section, which motivates us to show the details of our calculation here.

${ }^{14}$ To obtain the results in SM, one may simply set the right-handed neutrino terms to zero.
} 
and $d \Pi_{P} \equiv \prod_{i} d^{3} p_{i} /\left[(2 \pi)^{3} 2 E_{i}\right](2 \pi)^{4} \delta^{(4)}\left(\sum_{\text {in }} p_{-} \sum_{\text {out }} P\right)$ the usual definition of phase space and $E_{\nu}$ the energy of the (anti)neutrino produced in the decay process. In our setup, we find the only non-vanishing terms contributing to the production coefficients $p_{X L}$ are the $g_{22}$ and $h_{21}$ terms in eq. (A.1), whereas all the others vanish due to chiral flip. As a result, one only needs to calculate the following two integrals:

$$
I_{1} \equiv \int d \Pi_{P^{\prime}} A_{L}^{P} \bar{A}_{L}^{P}, \quad I_{2} \equiv \int d \Pi_{P^{\prime}} A_{h 21}^{P} \bar{A}_{L}^{P},
$$

where $A_{h 21}$ corresponds to the amplitude from the $h_{21}$ term in eq. (A.1). We find

$$
I_{1}=\mathcal{F} \int d \Pi_{P^{\prime}} 256\left(p_{1} \cdot p_{3}\right)\left(p_{2} \cdot p_{4}\right), \quad I_{2}=\mathcal{F} \int d \Pi_{P^{\prime}} 64 m_{e} m_{\mu}\left(p_{1} \cdot p_{4}\right),
$$

where $\mathcal{F}$ is an overall factor that will cancel eventually when one calculates the production coefficients. Therefore, the problem boils down to a three-body phase space integral, for which we will do in the following general form and start with the full phase space integral $d \Pi_{P}$ instead of $d \Pi_{P^{\prime}}$ :

$$
I \equiv \int d \Pi_{p}\left\langle\mathcal{M}^{2}\right\rangle, \quad \text { with }\left\langle\mathcal{M}^{2}\right\rangle=\left\{\begin{aligned}
256\left(p_{1} \cdot p_{3}\right)\left(p_{2} \cdot p_{4}\right), & \text { for } I_{1} \\
64 m_{e} m_{\mu}\left(p_{1} \cdot p_{4}\right), & \text { for } I_{2}
\end{aligned}\right.
$$

Writing explicitly,

$$
\begin{aligned}
I= & \int \frac{d^{3} p_{1}}{(2 \pi)^{3} 2 E_{1}} \frac{d^{3} p_{4}}{(2 \pi)^{3} 2 E_{4}} \frac{d^{3} p_{3}}{(2 \pi)^{3} 2 E_{3}}(2 \pi)^{4} \delta^{(4)}\left(p_{2}-p_{1}-p_{3}-p_{4}\right)\left\langle\mathcal{M}^{2}\right\rangle \\
= & \int \frac{d^{3} p_{1}}{(2 \pi)^{3} 2 E_{1}} \frac{d^{3} p_{4}}{(2 \pi)^{3} 2 E_{4}} \frac{d^{4} p_{3}}{(2 \pi)^{3}} \Theta\left(E_{3}\right) \delta\left(p_{3}^{2}-m_{e}^{2}\right)(2 \pi)^{4} \delta^{(4)}\left(p_{2}-p_{1}-p_{3}-p_{4}\right)\left\langle\mathcal{M}^{2}\right\rangle \\
= & \left.\frac{1}{(2 \pi)^{5}} \frac{1}{2^{2}} \int \frac{d^{3} p_{1}}{E 1} \frac{d^{3} p_{4}}{E 4} \Theta\left(E_{2}-E_{1}-E_{4}\right)\left\langle\mathcal{M}^{2}\right\rangle\right|_{p_{3} \rightarrow p_{2}-p_{1}-p_{4}} \\
& \times \delta\left(m_{\mu}^{2}-m_{e}^{2}-2 p_{2} \cdot p_{1}-2 p_{2} \cdot p_{4}+2 p_{1} \cdot p_{4}\right)
\end{aligned}
$$

Working in the rest frame of the decaying muon and performing the angular integrals, we arrive at

$$
\begin{aligned}
I= & \frac{1}{32 \pi^{3}} \int d E_{1} \int d E_{4} \Theta\left(m_{\mu}-E_{1}-E_{4}\right) \\
& \times\left.\left(\left.\left\langle\mathcal{M}^{2}\right\rangle\right|_{p_{3} \rightarrow p_{2}-p_{1}-p_{4}}\right)\right|_{\cos \theta=\frac{m_{\mu}^{2}-m_{e}^{2}-2 m_{\mu}\left(E_{1}+E_{4}\right)+2 E_{1} E_{4}}{2 E_{1} E_{4}},}
\end{aligned}
$$

where $\theta$ is the angle between the three-momenta $\vec{p}_{1}$ and $\vec{p}_{4}$, and $\Theta$ is the Heaviside function that ensures the positiveness of the electron's energy in the final state. Constraints on $E_{1,4}$ can be obtained by requiring

$$
m_{\mu}-E_{1}-E_{4}>0, \quad-1 \leq \cos \theta \leq 1,
$$

leading to

$$
\begin{aligned}
& \frac{m_{\mu}^{2}-m_{e}^{2}}{2 m_{\mu}}-E_{4} \leq E_{1} \leq \frac{m_{\mu}}{2}-\frac{m_{e}^{2}}{2\left(m_{\mu}-2 E_{4}\right)} \\
& \frac{m_{\mu}^{2}-m_{e}^{2}}{2 m_{\mu}}-E_{1} \leq E_{4} \leq \frac{m_{\mu}}{2}-\frac{m_{e}^{2}}{2\left(m_{\mu}-2 E_{1}\right)}
\end{aligned}
$$


Therefore, for neutrino production, one finally has

$$
I_{1,2}^{\nu}=\frac{d I}{d E_{1}}=\left.\frac{1}{32 \pi^{3}} \int_{E_{4}^{\min }}^{E_{4}^{\max }} d E_{4}\left(\left.\left\langle\mathcal{M}^{2}\right\rangle\right|_{p_{3} \rightarrow p_{2}-p_{1}-p_{4}}\right)\right|_{\cos \theta=\frac{m_{\mu}^{2}-m_{e}^{2}-2 m_{\mu}\left(E_{1}+E_{4}\right)+2 E_{1} E_{4}}{2 E_{1} E_{4}}},
$$

and, for antineutrino production,

$$
I_{1,2}^{\bar{\nu}}=\frac{d I}{d E_{4}}=\left.\frac{1}{32 \pi^{3}} \int_{E_{1}^{\min }}^{E_{1}^{\max }} d E_{1}\left(\left.\left\langle\mathcal{M}^{2}\right\rangle\right|_{p_{3} \rightarrow p_{2}-p_{1}-p_{4}}\right)\right|_{\cos \theta=\frac{m_{\mu}^{2}-m_{e}^{2}-2 m_{\mu}\left(E_{1}+E_{4}\right)+2 E_{1} E_{4}}{2 E_{1} E_{4}}},
$$

where $I_{1,2}^{\nu, \bar{\nu}}$ are obtained with appropriate $\left\langle\mathcal{M}^{2}\right\rangle$ given in eq. (A.8).

Finally, using eq. (A.5) and $I_{1,2}^{\nu, \bar{\nu}}$ derived above, we obtain ${ }^{15}$

$$
\begin{aligned}
& p_{L L}=1, \quad p_{h_{21} L}=\frac{3 m_{e} m_{\mu}\left(m_{\mu}-2 E_{\nu}\right)}{16 m_{\mu} E_{\nu}^{2}+6 m_{\mu}\left(m_{\mu}^{2}+m_{e}^{2}\right)-4 E_{\nu}\left(5 m_{\mu}^{2}+m_{e}^{2}\right)}, \\
& \bar{p}_{L L}=1, \quad \bar{p}_{h_{21} L}=\frac{m_{e}}{4\left(m_{\mu}-2 E_{\bar{\nu}}\right)},
\end{aligned}
$$

for neutrino and antineutrino production respectively, and thus the matching formulae can be written as

$$
\begin{aligned}
\epsilon_{\mu \beta}^{s} & =\left[g_{22}+\frac{3 m_{e} m_{\mu}\left(m_{\mu}-2 E_{\nu}\right)}{16 m_{\mu} E_{\nu}^{2}+6 m_{\mu}\left(m_{\mu}^{2}+m_{e}^{2}\right)-4 E_{\nu}\left(5 m_{\mu}^{2}+m_{e}^{2}\right)} h_{21}\right]_{\mu \beta}^{*}, \\
\epsilon_{e \beta}^{s} & =\left[g_{22}+\frac{m_{e}}{4\left(m_{\mu}-2 E_{\bar{\nu}}\right)} h_{21}\right]_{e \beta}^{*} .
\end{aligned}
$$

We checked that these results agree with those in ref. [20] after performing a small $m_{e}$ expansion and using the notation relations specified in eq. (A.3). ${ }^{16}$

Open Access. This article is distributed under the terms of the Creative Commons Attribution License (CC-BY 4.0), which permits any use, distribution and reproduction in any medium, provided the original author(s) and source are credited.

\section{References}

[1] Super-Kamiokande collaboration, Evidence for oscillation of atmospheric neutrinos, Phys. Rev. Lett. 81 (1998) 1562 [hep-ex/9807003] [INSPIRE].

[2] SNO collaboration, Direct evidence for neutrino flavor transformation from neutral current interactions in the Sudbury Neutrino Observatory, Phys. Rev. Lett. 89 (2002) 011301 [nucl-ex/0204008] [INSPIRE].

\footnotetext{
${ }^{15}$ We use $E_{\nu(\bar{\nu})}$ to replace $E_{1(4)}$ here to specify the general fact here that the production coefficients are (anti)neutrino-energy dependent. The difference in the matching formulae for neutrinos and antineutrinos results from the variable being integrated over or equivalently the feature of the amplitudes in eq. (A.8), as was also explained in ref. [20].

${ }^{16}$ Note that we have ignored the other flavor indices above compared with the results in ref. [20].
} 
[3] I. Esteban, M.C. Gonzalez-Garcia, M. Maltoni, T. Schwetz and A. Zhou, The fate of hints: updated global analysis of three-flavor neutrino oscillations, JHEP 09 (2020) 178 [arXiv:2007.14792] [INSPIRE].

[4] S. Davidson, C. Pena-Garay, N. Rius and A. Santamaria, Present and future bounds on nonstandard neutrino interactions, JHEP 03 (2003) 011 [hep-ph/0302093] [INSPIRE].

[5] J. Barranco, O.G. Miranda, C.A. Moura and J.W.F. Valle, Constraining non-standard interactions in $\nu_{e}$ e or $\bar{\nu}_{e}$ e scattering, Phys. Rev. D 73 (2006) 113001 [hep-ph/0512195] [INSPIRE].

[6] J. Barranco, O.G. Miranda, C.A. Moura and J.W.F. Valle, Constraining non-standard neutrino-electron interactions, Phys. Rev. D 77 (2008) 093014 [arXiv:0711.0698] [INSPIRE].

[7] D.V. Forero and M.M. Guzzo, Constraining nonstandard neutrino interactions with electrons, Phys. Rev. D 84 (2011) 013002 [INSPIRE].

[8] R. Adhikari, S. Chakraborty, A. Dasgupta and S. Roy, Non-standard interaction in neutrino oscillations and recent Daya Bay, T2K experiments, Phys. Rev. D 86 (2012) 073010 [arXiv: 1201.3047] [INSPIRE].

[9] I. Girardi, D. Meloni and S.T. Petcov, The Daya Bay and T2K results on $\sin ^{2} 2 \theta_{13}$ and Non-Standard Neutrino Interactions, Nucl. Phys. B 886 (2014) 31 [arXiv:1405.0416] [INSPIRE].

[10] A. Di Iura, I. Girardi and D. Meloni, Probing new physics scenarios in accelerator and reactor neutrino experiments, J. Phys. G 42 (2015) 065003 [arXiv: 1411.5330] [InSPIRE].

[11] J. Tang, Y. Zhang and Y.-F. Li, Probing Direct and Indirect Unitarity Violation in Future Accelerator Neutrino Facilities, Phys. Lett. B 774 (2017) 217 [arXiv:1708.04909] [INSPIRE].

[12] K.S. Babu, P.S.B. Dev, S. Jana and A. Thapa, Non-Standard Interactions in Radiative Neutrino Mass Models, JHEP 03 (2020) 006 [arXiv:1907.09498] [INSPIRE].

[13] T. Ohlsson, Status of non-standard neutrino interactions, Rept. Prog. Phys. 76 (2013) 044201 [arXiv: 1209.2710] [INSPIRE].

[14] P.S. Bhupal Dev et al., Neutrino Non-Standard Interactions: A Status Report, [INSPIRE].

[15] Y. Grossman, Nonstandard neutrino interactions and neutrino oscillation experiments, Phys. Lett. $B 359$ (1995) 141 [hep-ph/9507344] [INSPIRE].

[16] M.C. Gonzalez-Garcia, Y. Grossman, A. Gusso and Y. Nir, New CP-violation in neutrino oscillations, Phys. Rev. D 64 (2001) 096006 [hep-ph/0105159] [INSPIRE].

[17] T. Ohlsson and H. Zhang, Non-Standard Interaction Effects at Reactor Neutrino Experiments, Phys. Lett. B 671 (2009) 99 [arXiv:0809.4835] [INSPIRE].

[18] Y. Farzan and M. Tortola, Neutrino oscillations and Non-Standard Interactions, Front. in Phys. 6 (2018) 10 [arXiv:1710.09360] [INSPIRE].

[19] L. Wolfenstein, Neutrino Oscillations in Matter, Phys. Rev. D 17 (1978) 2369 [INSPIRE].

[20] A. Falkowski, M. González-Alonso and Z. Tabrizi, Consistent QFT description of non-standard neutrino interactions, JHEP 11 (2020) 048 [arXiv:1910.02971] [INSPIRE].

[21] T2K collaboration, Constraint on the matter-antimatter symmetry-violating phase in neutrino oscillations, Nature 580 (2020) 339 [Erratum ibid. 583 (2020) E16] [arXiv: 1910.03887] [INSPIRE]. 
[22] T2K collaboration, The T2K Experiment, Nucl. Instrum. Meth. A 659 (2011) 106 [arXiv: 1106.1238] [INSPIRE].

[23] NOvA collaboration, The NOvA Technical Design Report, FERMILAB-DESIGN-2007-01.

[24] NOvA collaboration, First Measurement of Neutrino Oscillation Parameters using Neutrinos and Antineutrinos by NOvA, Phys. Rev. Lett. 123 (2019) 151803 [arXiv: 1906.04907] [INSPIRE].

[25] DaYa BAy collaboration, Improved Measurement of Electron Antineutrino Disappearance at Daya Bay, Chin. Phys. C 37 (2013) 011001 [arXiv:1210.6327] [INSPIRE].

[26] DAYA BAY collaboration, Spectral measurement of electron antineutrino oscillation amplitude and frequency at Daya Bay, Phys. Rev. Lett. 112 (2014) 061801 [arXiv: 1310.6732] [INSPIRE].

[27] DaYA BAy collaboration, Measurement of the Electron Antineutrino Oscillation with 1958 Days of Operation at Daya Bay, Phys. Rev. Lett. 121 (2018) 241805 [arXiv:1809.02261] [INSPIRE].

[28] Double CHOOZ collaboration, Double CHOOZ: A search for the neutrino mixing angle theta(13), hep-ex/0606025 [INSPIRE].

[29] Double CHOOZ collaboration, Double CHOOZ $\theta_{13}$ measurement via total neutron capture detection, Nature Phys. 16 (2020) 558 [arXiv:1901.09445] [INSPIRE].

[30] RENO collaboration, Observation of Reactor Electron Antineutrino Disappearance in the RENO Experiment, Phys. Rev. Lett. 108 (2012) 191802 [arXiv:1204.0626] [INSPIRE].

[31] RENO collaboration, Measurement of Reactor Antineutrino Oscillation Amplitude and Frequency at RENO, Phys. Rev. Lett. 121 (2018) 201801 [arXiv:1806.00248] [INSPIRE].

[32] J. Aebischer, J. Kumar and D.M. Straub, Wilson: a Python package for the running and matching of Wilson coefficients above and below the electroweak scale, Eur. Phys. J. C 78 (2018) 1026 [arXiv:1804.05033] [INSPIRE].

[33] V. Cirigliano, J. Jenkins and M. Gonzalez-Alonso, Semileptonic decays of light quarks beyond the Standard Model, Nucl. Phys. B $\mathbf{8 3 0}$ (2010) 95 [arXiv:0908.1754] [InSPIRE].

[34] M. González-Alonso, J. Martin Camalich and K. Mimouni, Renormalization-group evolution of new physics contributions to (semi)leptonic meson decays, Phys. Lett. B $\mathbf{7 7 2}$ (2017) 777 [arXiv:1706.00410] [INSPIRE].

[35] P. Huber, M. Lindner and W. Winter, Simulation of long-baseline neutrino oscillation experiments with GLoBES (General Long Baseline Experiment Simulator), Comput. Phys. Commun. 167 (2005) 195 [hep-ph/0407333] [INSPIRE].

[36] P. Huber, J. Kopp, M. Lindner, M. Rolinec and W. Winter, New features in the simulation of neutrino oscillation experiments with GLoBES 3.0: General Long Baseline Experiment Simulator, Comput. Phys. Commun. 177 (2007) 432 [hep-ph/0701187] [INSPIRE].

[37] J. Kopp, The new physics tool, https://www.mpi-hd.mpg.de/personalhomes/globes/tools/snu-1.0.pdf, (2010).

[38] B. Pontecorvo, Mesonium and anti-mesonium, Sov. Phys. JETP 6 (1957) 429 [InSPIRE].

[39] B. Pontecorvo, Inverse beta processes and nonconservation of lepton charge, Sov. Phys. JETP 7 (1958) 172 [INSPIRE]. 
[40] Z. Maki, M. Nakagawa, Y. Ohnuki and S. Sakata, A unified model for elementary particles, Prog. Theor. Phys. 23 (1960) 1174 [InSPIRE].

[41] Z. Maki, M. Nakagawa and S. Sakata, Remarks on the unified model of elementary particles, Prog. Theor. Phys. 28 (1962) 870 [INSPIRE].

[42] B. Pontecorvo, Neutrino Experiments and the Problem of Conservation of Leptonic Charge, Sov. Phys. JETP 26 (1968) 984 [inSPIRE].

[43] S.P. Mikheyev and A.Y. Smirnov, Resonance Amplification of Oscillations in Matter and Spectroscopy of Solar Neutrinos, Sov. J. Nucl. Phys. 42 (1985) 913 [inSPIRE].

[44] S. Antusch, C. Biggio, E. Fernandez-Martinez, M.B. Gavela and J. Lopez-Pavon, Unitarity of the Leptonic Mixing Matrix, JHEP 10 (2006) 084 [hep-ph/0607020] [INSPIRE].

[45] D. Choudhury, K. Ghosh and S. Niyogi, Probing nonstandard neutrino interactions at the LHC Run II, Phys. Lett. B 784 (2018) 248 [arXiv:1801.01513] [INSPIRE].

[46] M. Masud, S. Roy and P. Mehta, Correlations and degeneracies among the NSI parameters with tunable beams at DUNE, Phys. Rev. D 99 (2019) 115032 [arXiv:1812.10290] [InSPIRE].

[47] A. Friedland, M.L. Graesser, I.M. Shoemaker and L. Vecchi, Probing Nonstandard Standard Model Backgrounds with LHC Monojets, Phys. Lett. B 714 (2012) 267 [arXiv:1111.5331] [INSPIRE].

[48] S. Pandey, S. Karmakar and S. Rakshit, Strong constraints on non-standard neutrino interactions: LHC vs. IceCube, JHEP 11 (2019) 046 [arXiv: 1907.07700] [INSPIRE].

[49] K.S. Babu, D. Gonçalves, S. Jana and P.A.N. Machado, Neutrino Non-Standard Interactions: Complementarity Between LHC and Oscillation Experiments, Phys. Lett. B 815 (2021) 136131 [arXiv:2003.03383] [INSPIRE].

[50] D. Liu, C. Sun and J. Gao, Constraints on neutrino non-standard interactions from LHC data with large missing transverse momentum, JHEP 02 (2021) 033 [arXiv:2009.06668] [INSPIRE].

[51] U.K. Dey, N. Nath and S. Sadhukhan, Charged Higgs effects in IceCube: PeV events and NSIs, arXiv:2010.05797 [INSPIRE].

[52] I. Bischer and W. Rodejohann, General neutrino interactions from an effective field theory perspective, Nucl. Phys. B 947 (2019) 114746 [arXiv:1905.08699] [INSPIRE].

[53] C.C. Chang et al., A per-cent-level determination of the nucleon axial coupling from quantum chromodynamics, Nature $\mathbf{5 5 8}$ (2018) 91 [arXiv:1805.12130] [INSPIRE].

[54] R. Gupta, Y.-C. Jang, B. Yoon, H.-W. Lin, V. Cirigliano and T. Bhattacharya, Isovector Charges of the Nucleon from 2+1+1-flavor Lattice QCD, Phys. Rev. D 98 (2018) 034503 [arXiv: 1806.09006] [INSPIRE].

[55] Flavour Lattice Averaging Group collaboration, FLAG Review 2019: Flavour Lattice Averaging Group (FLAG), Eur. Phys. J. C 80 (2020) 113 [arXiv:1902.08191] [INSPIRE].

[56] M. González-Alonso and J. Martin Camalich, Isospin breaking in the nucleon mass and the sensitivity of $\beta$ decays to new physics, Phys. Rev. Lett. 112 (2014) 042501 [arXiv: 1309.4434] [INSPIRE].

[57] A. Falkowski, M. González-Alonso and Z. Tabrizi, Reactor neutrino oscillations as constraints on Effective Field Theory, JHEP 05 (2019) 173 [arXiv: 1901.04553] [INSPIRE]. 
[58] A.C. Hayes and P. Vogel, Reactor Neutrino Spectra, Ann. Rev. Nucl. Part. Sci. 66 (2016) 219 [arXiv: 1605. 02047] [INSPIRE].

[59] E.E. Jenkins, A.V. Manohar and P. Stoffer, Low-Energy Effective Field Theory below the Electroweak Scale: Anomalous Dimensions, JHEP 01 (2018) 084 [arXiv:1711.05270] [INSPIRE].

[60] J. Terol-Calvo, M. Tórtola and A. Vicente, High-energy constraints from low-energy neutrino nonstandard interactions, Phys. Rev. D 101 (2020) 095010 [arXiv: 1912.09131] [InSPIRE].

[61] A. Falkowski, M. González-Alonso and K. Mimouni, Compilation of low-energy constraints on 4-fermion operators in the SMEFT, JHEP 08 (2017) 123 [arXiv:1706.03783] [INSPIRE].

[62] E.E. Jenkins, A.V. Manohar and P. Stoffer, Low-Energy Effective Field Theory below the Electroweak Scale: Operators and Matching, JHEP 03 (2018) 016 [arXiv:1709.04486] [INSPIRE].

[63] S. Weinberg, Baryon and Lepton Nonconserving Processes, Phys. Rev. Lett. 43 (1979) 1566 [INSPIRE].

[64] M.K. Gaillard, The Effective One Loop Lagrangian With Derivative Couplings, Nucl. Phys. B 268 (1986) 669 [INSPIRE].

[65] O. Cheyette, Effective Action for the Standard Model With Large Higgs Mass, Nucl. Phys. B 297 (1988) 183 [INSPIRE].

[66] B. Henning, X. Lu and H. Murayama, How to use the Standard Model effective field theory, JHEP 01 (2016) 023 [arXiv: 1412.1837] [INSPIRE].

[67] W. Skiba, Effective Field Theory and Precision Electroweak Measurements, in Theoretical Advanced Study Institute in Elementary Particle Physics: Physics of the Large and the Small, (2011), pp. 5-70, DOI [arXiv:1006.2142] [INSPIRE].

[68] R. Alonso, E.E. Jenkins, A.V. Manohar and M. Trott, Renormalization Group Evolution of the Standard Model Dimension Six Operators III: Gauge Coupling Dependence and Phenomenology, JHEP 04 (2014) 159 [arXiv: 1312. 2014] [INSPIRE].

[69] E.E. Jenkins, A.V. Manohar and M. Trott, Renormalization Group Evolution of the Standard Model Dimension Six Operators I: Formalism and lambda Dependence, JHEP 10 (2013) 087 [arXiv: 1308.2627] [INSPIRE].

[70] E.E. Jenkins, A.V. Manohar and M. Trott, Renormalization Group Evolution of the Standard Model Dimension Six Operators II: Yukawa Dependence, JHEP 01 (2014) 035 [arXiv: 1310.4838] [INSPIRE].

[71] Neutrino 2020, https://conferences.fnal.gov/nu2020/, (2020).

[72] T2K collaboration, T2K measurements of muon neutrino and antineutrino disappearance using $3.13 \times 10^{21}$ protons on target, Phys. Rev. D 103 (2021) L011101 [arXiv:2008.07921] [INSPIRE].

[73] T2K collaboration, Most recent T2K results on CPV in neutrino sector, Nuovo Cim. C 42 (2020) 246 [INSPIRE].

[74] G.L. Fogli, E. Lisi, A. Marrone, D. Montanino and A. Palazzo, Getting the most from the statistical analysis of solar neutrino oscillations, Phys. Rev. D 66 (2002) 053010 [hep-ph/0206162] [INSPIRE].

[75] NuFIT collaboration, Nufit 5.0, http://www.nu-fit.org/?q=node/211, (2020). 
[76] I. Esteban, M.C. Gonzalez-Garcia, M. Maltoni, I. Martinez-Soler and J. Salvado, Updated constraints on non-standard interactions from global analysis of oscillation data, JHEP 08 (2018) 180 [Addendum ibid. 12 (2020) 152] [arXiv: 1805.04530] [INSPIRE].

[77] V. Gherardi, D. Marzocca and E. Venturini, Matching scalar leptoquarks to the SMEFT at one loop, JHEP 07 (2020) 225 [Erratum ibid. 01 (2021) 006] [arXiv:2003.12525] [INSPIRE].

[78] J. Aebischer, A. Crivellin, M. Fael and C. Greub, Matching of gauge invariant dimension-six operators for $b \rightarrow s$ and $b \rightarrow c$ transitions, JHEP 05 (2016) 037 [arXiv:1512.02830] [INSPIRE].

[79] CMS collaboration, Search for singly and pair-produced leptoquarks coupling to third-generation fermions in proton-proton collisions at $\sqrt{s}=13 \mathrm{TeV}$, Tech. Rep. CMS-PAS-EXO-19-015, CERN, Geneva (2020).

[80] ATLAS collaboration, Searches for third-generation scalar leptoquarks in $\sqrt{s}=13 \mathrm{TeV} p p$ collisions with the ATLAS detector, JHEP 06 (2019) 144 [arXiv: 1902.08103] [INSPIRE].

[81] K. Fuyuto, M. Ramsey-Musolf and T. Shen, Electric Dipole Moments from CP-Violating Scalar Leptoquark Interactions, Phys. Lett. B $\mathbf{7 8 8}$ (2019) 52 [arXiv:1804.01137] [InSPIRE].

[82] J. Elias-Miró, C. Grojean, R.S. Gupta and D. Marzocca, Scaling and tuning of $E W$ and Higgs observables, JHEP 05 (2014) 019 [arXiv: 1312.2928] [INSPIRE].

[83] P.B. Denton, J. Gehrlein and R. Pestes, CP - Violating Neutrino Nonstandard Interactions in Long-Baseline-Accelerator Data, Phys. Rev. Lett. 126 (2021) 051801 [arXiv:2008.01110] [INSPIRE].

[84] S.S. Chatterjee and A. Palazzo, Nonstandard Neutrino Interactions as a Solution to the

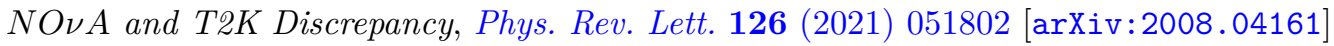
[INSPIRE].

[85] S.K. Agarwalla, S.S. Chatterjee and A. Palazzo, Octant of $\theta_{23}$ in danger with a light sterile neutrino, Phys. Rev. Lett. 118 (2017) 031804 [arXiv:1605.04299] [INSPIRE].

[86] K. Mursula and F. Scheck, Analysis of Leptonic Charged Weak Interactions, Nucl. Phys. B 253 (1985) 189.

[87] F. Scheck, Muon Physics, Phys. Rept. 44 (1978) 187 [InSPIRE].

[88] F. Scheck, Leptons, hadrons and nuclei, North-holland, Amsterdam, Netherlands (1983), [INSPIRE]. 